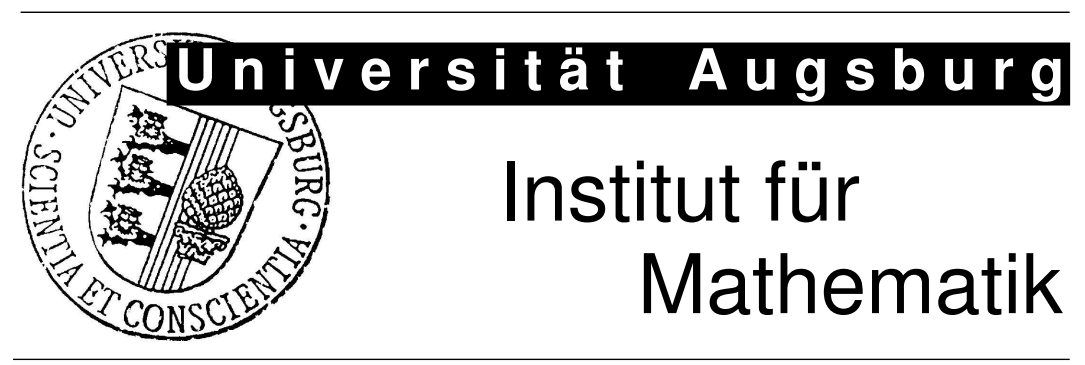

Thomas Franke, Ronald H.W. Hoppe, Christopher Linsenmann, Achim Wixforth

Projection Based Model Reduction for Optimal Design of the Time-Dependent Stokes System 


\section{Impressum:}

\section{Herausgeber:}

Institut für Mathematik

Universität Augsburg

86135 Augsburg

http://www.math.uni-augsburg.de/pages/de/forschung/preprints.shtml

\section{ViSdP:}

Ronald H.W. Hoppe

Institut für Mathematik

Universität Augsburg

86135 Augsburg

Preprint: Sämtliche Rechte verbleiben den Autoren (C) 2010 


\title{
Projection Based Model Reduction for Optimal Design of the Time-Dependent Stokes System
}

\author{
T. Franke, R.H.W. Hoppe, C. Linsenmann and A. Wixforth
}

\begin{abstract}
The optimal design of structures and systems described by partial differential equations (PDEs) often gives rise to large-scale optimization problems, in particular if the underlying system of PDEs represents a multi-scale, multi-physics problem. Therefore, reduced order modeling techniques such as balanced truncation model reduction, proper orthogonal decomposition, or reduced basis methods are used to significantly decrease the computational complexity while maintaining the desired accuracy of the approximation. In particular, we are interested in such shape optimization problems where the design issue is restricted to a relatively small portion of the computational domain. In this case, it appears to be natural to rely on a full order model only in that specific part of the domain and to use a reduced order model elsewhere. A convenient methodology to realize this idea consists in a suitable combination of domain decomposition techniques and balanced truncation model reduction. We will consider such an approach for shape optimization problems associated with the time-dependent Stokes system and derive explicit error bounds for the modeling error.

As an application in life sciences, we will be concerned with the optimal design of capillary barriers as part of a network of microchannels and reservoirs on microfluidic biochips that are used in clinical diagnostics, pharmacology, and forensics for high-throughput screening and hybridization in genomics and protein profiling in proteomics.
\end{abstract}

Mathematics Subject Classification (2000). Primary 65K10; Secondary 49M05; 49M15; 65M55; 65M60; 76Z99; 90C06.

Keywords. projection based model reduction, shape optimization, time-dependent Stokes system; domain decomposition, balanced truncation, microfluidic biochips.

The authors acknowledge support by the German National Science Foundation DFG within the DFG Priority Program SPP 1253 'Optimierung mit partiellen Differentialgleichungen'. 


\section{Introduction}

The numerical solution of optimization problems governed by time-dependent partial differential equations (PDEs) can be computationally very expensive with respect to both storage and CPU times. Therefore, serious attempts have been undertaken to achieve a significant reduction of the computational costs based on Reduced Order Models (ROMs). ROMs determine a subspace that contains the 'essential' dynamics of the time-dependent PDEs and project these PDEs onto the subspace. If the subspace is small, the original PDEs in the optimization problem can be replaced by a small system of ordinary differential equations and the resulting approximate optimization problem can be solved efficiently. Among the most commonly used ROM techniques are balanced truncation model reduction, Krylov subspace methods, proper orthogonal decomposition (POD), and reduced basis methods (see, e.g., the books and survey articles $[8,10,11,12,20,41,47]$ and the references therein).

Among the challenges one has to overcome when one wants to apply ROM techniques for optimization problems are the efficient computation of ROMs for use in optimization and the derivation of error estimates for ROMs. Some aspects of these questions have been addressed in $[3,4,15,16,17,29,30,31,34,35,36$, $38,39,44,45,50,51,52]$. In most of these applications, estimates for the error between the solution of the original optimization problem and the optimization problem governed by the reduced order model are not available; if they exist, then only for a restricted class of problems. Different types of error estimates for some ROM approaches have been discussed in, e.g., [3, 4, 23, 24, 27, 29, 30, 43, 50].

In this contribution, we will apply balanced truncation model reduction (BTMR) to the optimal design of systems whose operational behavior is described by the time-dependent Stokes equations. Since BTMR is essentially restricted to linear time-invariant systems, whereas optimal design problems are genuinely nonlinear in nature due to the nonlinear dependence on the design variables, we consider a semi-discretization in space of the time-dependent Stokes equations (Section 2) and focus on such problems where the design is restricted to a relatively small part of the computational domain. In particular, we use a combination of domain decomposition and BTMR in the sense that we keep the full order model for the subdomain subject to the design and use BTMR for the rest of the domain (Sections 3 and 4). It turns out that the reduced optimality system represents the necessary optimality conditions of a reduced optimal design problem featuring a reduced objective functional. This observation is the key for an a priori analysis of the modeling error (Section 5). It should be emphasized that the full order model refers to the semi-discretized Stokes system. Hence, the error induced by the discretization in space is not taken into account. The main result states that under some assumptions on the objective functional the error between the optimal design for the full order model and the reduced order model is bounded by a constant times the sum of those Hankel singular values in the associated singular value decomposition involving the controllability and observability Gramians that 
are not used for the derivation of the ROM.

The combination of domain decomposition and balanced truncation model reduction (DDBTMR) is applied to the optimal design of surface acoustic wave driven microfluidic biochips which are used for high-throughput screening and hybridization in genomics and protein profiling in proteomics. In particular, we consider the shape optimization of capillary barriers between the channels on top of the biochips and reservoirs where the chemical analysis of the probes is performed (Section 6).

\section{Optimal design of the Stokes equations and semi-discretization in space}

Let $\Omega(\theta) \subset \mathbb{R}^{2}$ be a bounded domain that depends on design variables $\theta=$ $\left(\theta_{1}, \cdots, \theta_{d}\right)^{T} \in \Theta$, where $\theta_{i}, 1 \leq i \leq d$, are the Bézier control points of a Bézier curve representation of the boundary $\partial \Omega(\theta)$ and $\Theta$ stands for the convex set

$$
\Theta:=\left\{\theta_{i} \in \mathbb{R} \mid \theta_{\text {min }}^{(i)} \leq \theta_{i} \leq \theta_{\text {max }}^{(i)}, 1 \leq i \leq d\right\}
$$

with $\theta_{\min }^{(i)}, \theta_{\max }^{(i)}, 1 \leq i \leq d$, being given. We assume that the boundary $\partial \Omega(\theta)$ consists of an inflow boundary $\Gamma_{i n}(\theta)$, an outflow boundary $\Gamma_{\text {out }}(\theta)$, and a lateral boundary $\Gamma_{\text {lat }}(\theta)$ such that $\partial \Omega(\theta)=\bar{\Gamma}_{\text {in }}(\theta) \cup \bar{\Gamma}_{\text {out }}(\theta) \cup \bar{\Gamma}_{\text {lat }}(\theta), \Gamma_{\text {in }}(\theta) \cap \Gamma_{\text {out }}(\theta) \cap$ $\Gamma_{\text {lat }}(\theta)=\emptyset$. We set $Q(\theta):=\Omega(\theta) \times(0, T), \Sigma(\theta):=\partial \Omega(\theta) \times(0, T), \Sigma_{\text {in }}(\theta):=$ $\Gamma_{i n}(\theta) \times(0, T), \Sigma_{l a t}(\theta):=\Gamma_{l a t}(\theta) \times(0, T), T>0$. Denoting by $v=\left(v_{1}, v_{2}\right)^{T}$ and $p$ the velocity and the pressure of a fluid with viscosity $\nu$ in $\bar{\Omega}(\theta)$ and by $\ell(v, p, x, t, \theta)$ a given function of the velocity, the pressure, the independent variables $x, t$, and the design variable $\theta$, we consider optimal design problems associated with the time-dependent Stokes system of the form

$$
\inf _{\theta \in \Theta} J(\theta):=\frac{1}{2} \int_{0}^{T} \int_{\Omega(\theta)} \ell(v, p, x, t, \theta) d x d t,
$$

subject to the Stokes flow

$$
\begin{aligned}
\frac{\partial v}{\partial t}-\nu \Delta v+\nabla p & =f \quad \text { in } Q(\theta) \\
\nabla \cdot v & =0 \quad \text { in } Q(\theta) \\
v & =v^{i n} \quad \text { on } \Sigma_{\text {in }}(\theta), \\
v & =0 \quad \text { on } \Sigma_{\text {lat }}(\theta) \\
(\nu \nabla v-p I) n & =0 \quad \text { on } \Sigma_{\text {out }}(\theta) \\
v(\cdot, 0) & =v^{(0)} \quad \text { in } \Omega(\theta)
\end{aligned}
$$

where $f$ is a given forcing term, $v^{\text {in }}$ stands for a prescribed velocity at the inflow boundary, $n$ in (2.1f) is the unit exterior normal, and $v^{(0)}$ is the velocity distribution at initial time $t=0$ with $\nabla \cdot v^{(0)}=0$. 
The discretization in space and time of the optimality system associated with (2.1a)-(2.1g) gives rise to a large-scale nonlinear optimization problem whose numerical solution requires considerable computational efforts, even if efficient solvers such as those based on 'all-at-once methods' are used. A significant reduction of the computational complexity can be achieved by projection based model reduction applied to a semi-discretization in space of the time-dependent Stokes equations, e.g., by stable continuous elements such as the Taylor-Hood P2-P1 element with respect to a simplicial triangulation of the spatial domain (cf.,e.g., [13, 14, 21]). Then, the the semi-discrete optimization problem reads

$$
\inf _{\theta \in \Theta} \mathbf{J}(\theta):=\int_{0}^{T} \ell(\mathbf{v}, \mathbf{p}, \theta, t) d t .
$$

The integrand $\boldsymbol{\ell}$ in (2.2a) stems from the semidiscretization of the inner integral in $(2.1 \mathrm{a})$, and the pair $(\mathbf{v}, \mathbf{p})$ is assumed to solve the Hessenberg index 2 system

$$
\begin{aligned}
\left(\begin{array}{cc}
\mathbf{M}(\theta) & 0 \\
0 & 0
\end{array}\right) \frac{d}{d t}\left(\begin{array}{c}
\mathbf{v}(t) \\
\mathbf{p}(t)
\end{array}\right)= & -\left(\begin{array}{cc}
\mathbf{A}(\theta) & \mathbf{B}^{T}(\theta) \\
\mathbf{B}(\theta) & 0
\end{array}\right)\left(\begin{array}{c}
\mathbf{v}(t) \\
\mathbf{p}(t)
\end{array}\right) \\
& +\left(\begin{array}{c}
\mathbf{K}(\theta) \\
\mathbf{L}(\theta)
\end{array}\right) \mathbf{f}(t) \quad, \quad t \in(0, T] \\
\mathbf{M}(\theta) \mathbf{v}(0)= & \mathbf{v}^{0}
\end{aligned}
$$

where $\mathbf{M}(\theta), \mathbf{A}(\theta) \in \mathbb{R}^{n \times n}$ stand for the mass and stiffness matrix, $\mathbf{B}(\theta) \in \mathbb{R}^{m \times n}$, $m<n$, refers to the discrete divergence operator, and $\mathbf{K}(\theta) \in \mathbb{R}^{n \times k}, \mathbf{L}(\theta) \in$ $\mathbb{R}^{m \times k}, \mathbf{f}(t) \in \mathbb{R}^{k}, t \in(0, T)$.

Under some assumptions on the matrices $\mathbf{M}(\theta), \mathbf{A}(\theta)$, and $\mathbf{B}(\theta)$, which are satisfied when using stable continuous elements, we can show continuous dependence of the solution of $(2.2 \mathrm{~b}),(2.2 \mathrm{c})$ on the data. This result will play a significant role in the a priori analysis of the modeling error in Section 5. For ease of notation we drop the dependence on the design variable $\theta$.

Theorem 2.1. Assume that $\mathbf{M} \in \mathbb{R}^{n \times n}$ is symmetric positive definite, $\mathbf{A} \in \mathbb{R}^{n \times n}$ is symmetric positive definite on $\operatorname{Ker} \mathbf{B}$, i.e.,

$$
\mathbf{v}^{T} \mathbf{A} \mathbf{v} \geq \alpha\|\mathbf{v}\|^{2} \quad, \quad \mathbf{v} \in \operatorname{Ker} \mathbf{B},
$$

and $\mathbf{B} \in \mathbb{R}^{m \times n}$ has full row rank $m$. Then, there exist positive constants $C_{1}$ and $\mathrm{C}_{2}$ such that

$$
\left(\begin{array}{c}
\|\mathbf{v}\|_{L^{2}} \\
\|\mathbf{p}\|_{L^{2}}
\end{array}\right) \leq C_{1}\left\|\mathbf{v}^{0}\right\|+C_{2}\left(\begin{array}{c}
\|\mathbf{f}\|_{\mathbf{L}^{2}} \\
\|\mathbf{f}\|_{L^{2}}+\left\|\frac{d}{d t} \mathbf{f}\right\|_{L^{2}}
\end{array}\right) .
$$

Proof. We introduce $\boldsymbol{\Pi}:=\mathbf{I}-\mathbf{B}^{T}\left(\mathbf{B M}^{-1} \mathbf{B}^{T}\right)^{-1} \mathbf{B M}^{-1}$ as an oblique projection onto Ker $\mathbf{B}^{T}$ along $\operatorname{Im} \mathbf{B}$ and split $\mathbf{v}(t)=\mathbf{v}_{H}(t)+\mathbf{v}_{P}(t)$, where $\mathbf{v}_{H}(t) \in$ Ker $\mathbf{B}$ and

$$
\mathbf{v}_{P}(t):=\mathbf{M}^{-1} \mathbf{B}^{T}\left(\mathbf{B M}^{-1} \mathbf{B}^{T}\right)^{-1} \mathbf{L} \mathbf{f}(t)
$$


is a particular solution of the second equation of the semi-discrete Stokes system $(2.2 \mathrm{~b}),(2.2 \mathrm{c})$. The semi-discrete Stokes system transforms to

$$
\begin{aligned}
& \boldsymbol{\Pi \mathbf { M }} \boldsymbol{\Pi}^{T} \frac{d}{d t} \mathbf{v}_{H}(t)=-\boldsymbol{\Pi} \mathbf{A} \boldsymbol{\Pi}^{T} \mathbf{v}_{H}(t)+\boldsymbol{\Pi} \tilde{\mathbf{K}} \mathbf{f}(t) \quad, \quad t \in(0, T], \\
& \Pi \mathbf{\Pi M} \boldsymbol{\Pi}^{T} \mathbf{v}_{H}(0)=\boldsymbol{\Pi} \mathbf{v}^{0} .
\end{aligned}
$$

The pressure $\mathbf{p}$ can be recovered according to

$$
\mathbf{p}(t)=\left(\mathbf{B} \mathbf{M}^{-1} \mathbf{B}^{T}\right)^{-1}\left(\mathbf{B} \mathbf{M}^{-1}\left(-\mathbf{A} \mathbf{v}_{H}(t)+\widetilde{\mathbf{K}} \mathbf{f}(t)\right)-\mathbf{L} \frac{d}{d t} \mathbf{f}(t)\right),
$$

where $\widetilde{\mathbf{K}}:=\mathbf{K}-\mathbf{A} \mathbf{M}^{-1} \mathbf{B}^{T}\left(\mathbf{B} \mathbf{M}^{-1} \mathbf{B}^{T}\right)^{-1} \mathbf{L}$. The assertion follows from Gronwall's lemma applied to (2.6a),(2.6b) and from $(2.5),(2.7)$.

\section{Balanced truncation model reduction of the semi-discretized Stokes optimality system}

We assume that the integrand $\boldsymbol{\ell}$ in $(2.2 \mathrm{a})$ is of the form

$$
\boldsymbol{\ell}(\mathbf{v}, \mathbf{p}, \theta, t):=\frac{1}{2}|\mathbf{C}(\theta) \mathbf{v}(t)+\mathbf{D}(\theta) \mathbf{p}(t)+\mathbf{F}(\theta) \mathbf{f}(t)-\mathbf{d}(t)|^{2}
$$

where $\mathbf{C}(\theta) \in \mathbb{R}^{q \times n}$ and $\mathbf{D}(\theta) \in \mathbb{R}^{q \times m}$ are observation matrices, $\mathbf{F}(\theta) \in \mathbb{R}^{q \times k}$, is a feedthrough matrix, and $\mathbf{d}(t) \in \mathbb{R}^{q}, t \in(0, T)$. Dropping again the dependence on $\theta$ for ease of notation, the semi-discretized Stokes optimality system consists of the state equations

$$
\begin{aligned}
\left(\begin{array}{cc}
\mathbf{M} & \mathbf{0} \\
\mathbf{0} & \mathbf{0}
\end{array}\right) \frac{d}{d t}\left(\begin{array}{c}
\mathbf{v}(t) \\
\mathbf{p}(t)
\end{array}\right) & =-\left(\begin{array}{cc}
\mathbf{A} & \mathbf{B}^{T} \\
\mathbf{B} & \mathbf{0}
\end{array}\right)\left(\begin{array}{c}
\mathbf{v}(t) \\
\mathbf{p}(t)
\end{array}\right)+\left(\begin{array}{c}
\mathbf{K} \\
\mathbf{L}
\end{array}\right) \mathbf{f}(t) \\
\mathbf{z}(t) & =\mathbf{C} \mathbf{v}(t)+\mathbf{D} \mathbf{p}(t)+\mathbf{F} \mathbf{f}(t) \\
\mathbf{M v}(0) & =\mathbf{v}^{0}
\end{aligned}
$$

and the adjoint equations

$$
\begin{aligned}
-\left(\begin{array}{cc}
\mathbf{M} & \mathbf{0} \\
\mathbf{0} & \mathbf{0}
\end{array}\right) \frac{d}{d t}\left(\begin{array}{c}
\boldsymbol{\lambda}(t) \\
\boldsymbol{\kappa}(t)
\end{array}\right) & =-\left(\begin{array}{cc}
\mathbf{A} & \mathbf{B}^{T} \\
\mathbf{B} & \mathbf{0}
\end{array}\right)\left(\begin{array}{c}
\boldsymbol{\lambda}(t) \\
\boldsymbol{\kappa}(t)
\end{array}\right)+\left(\begin{array}{c}
\mathbf{C}^{T} \\
\mathbf{D}^{T}
\end{array}\right) \mathbf{z}(t) \\
\mathbf{q}(t) & =\mathbf{K}^{T} \boldsymbol{\lambda}(t)+\mathbf{L}^{T} \boldsymbol{\kappa}(t)+\mathbf{F}^{T} \mathbf{z}(t) \\
\mathbf{M} \boldsymbol{\lambda}(T) & =\boldsymbol{\lambda}^{(T)}
\end{aligned}
$$

For the realization of the BTMR, we compute the controllability and observability Gramians $\mathbf{P}, \mathbf{Q} \in \mathbb{R}^{n \times n}$ as the solutions of the matrix Lyapunov equations

$$
\begin{aligned}
& \overline{\mathbf{A}} \mathbf{P} \overline{\mathbf{M}}+\overline{\mathbf{M}} \mathbf{P} \overline{\mathbf{A}}+\overline{\mathbf{K}} \overline{\mathbf{K}}^{T}=0, \\
& \overline{\mathbf{A}} \mathbf{Q} \overline{\mathbf{M}}+\overline{\mathbf{M}} \mathbf{Q} \overline{\mathbf{A}}+\overline{\mathbf{C}}^{T} \overline{\mathbf{C}}=0,
\end{aligned}
$$

where $\overline{\mathbf{A}}:=\Pi \mathbf{A} \Pi^{T}, \overline{\mathbf{M}}:=\Pi \mathbf{M} \boldsymbol{\Pi}^{T}, \overline{\mathbf{K}}:=\Pi \widetilde{\mathbf{K}}$, and $\overline{\mathbf{C}}:=\Pi \widetilde{\mathbf{C}}, \widetilde{\mathbf{C}}:=\mathbf{C}-$ $\mathbf{F}\left(\mathbf{B M}^{-1} \mathbf{B}^{T}\right)^{-1} \mathbf{B M}^{-1} \mathbf{A}$. The Lyapunov equations (3.4a),(3.4b) can be solved 
approximately by multishift ADI techniques (cf., e.g., [8]). We factorize $\mathbf{P}=$ $\mathbf{U U}^{T}, \mathbf{Q}=\mathbf{E E}^{T}$ and perform the singular value decomposition

$$
\mathbf{U}^{T} \mathbf{M E}=\mathbf{Z S}_{n} \mathbf{Y}^{T}, \mathbf{S}_{n}:=\operatorname{diag}\left(\sigma_{1}, \cdots, \sigma_{n}\right), \sigma_{i}>\sigma_{i+1}, 1 \leq i \leq n-1 .
$$

We compute $\mathbf{V}, \mathbf{W}$ according to

$$
\mathbf{V}:=\mathbf{U Z}_{p} \mathbf{S}_{p}^{-1 / 2} \quad, \quad \mathbf{W}:=\mathbf{E Y}_{p} \mathbf{S}_{p}^{-1 / 2},
$$

where $1 \leq p \leq n$ is chosen such that $\sigma_{p+1}<\tau \sigma_{1}$ for some threshold $\tau>0$ and $\mathbf{Y}_{p}, \mathbf{Z}_{p}$ are the matrices built by the leading $p$ columns of $\mathbf{Y}, \mathbf{Z}$.

The projection matrices satisfy

$$
\mathbf{V}=\mathbf{\Pi}^{T} \mathbf{V} \quad, \quad \mathbf{W}=\mathbf{\Pi}^{T} \mathbf{W} \quad, \quad \mathbf{W}^{T} \mathbf{M V}=\mathbf{I} .
$$

Multiplying the state equations by $\mathbf{W}^{T}$ and the adjoint equations by $\mathbf{V}^{T}$ results in a reduced order optimality system, where the reduced order state equations turn out to be

$$
\begin{aligned}
\frac{d}{d t} \widehat{\mathbf{v}}_{H}(t) & =-\widehat{\mathbf{A}} \widehat{\mathbf{v}}_{H}(t)+\widehat{\mathbf{K}} \mathbf{f}(t) \quad, \quad t \in(0, T], \\
\widehat{\mathbf{z}}(t) & =\widehat{\mathbf{C}} \widehat{\mathbf{v}}_{H}(t)+\widehat{\mathbf{G}} \mathbf{f}(t)-\widehat{\mathbf{H}} \frac{d}{d t} \mathbf{f}(t) \quad, \quad t \in(0, T], \\
\widehat{\mathbf{v}}_{H}(0) & =\widehat{\mathbf{v}}_{H}^{0},
\end{aligned}
$$

whereas the reduced order adjoint state equations are given according to

$$
\begin{aligned}
-\frac{d}{d t} \widehat{\boldsymbol{\lambda}}_{H}(t) & =-\widehat{\mathbf{A}}^{T} \widehat{\lambda}_{H}(t)+\widehat{\mathbf{C}}^{T} \widehat{\mathbf{z}}(t) \quad, \quad t \in[0, T), \\
\widehat{\mathbf{q}}(t) & =\widehat{\mathbf{K}}^{T} \widehat{\boldsymbol{\lambda}}_{H}(t)+\widehat{\mathbf{G}}^{T} \widehat{\mathbf{z}}(t)-\widehat{\mathbf{H}} \frac{d}{d t} \widehat{\mathbf{z}}(t) \quad, \quad t \in[0, T), \\
\widehat{\boldsymbol{\lambda}}_{H}(T) & =\widehat{\boldsymbol{\lambda}}^{(T)},
\end{aligned}
$$

with appropriately defined $\widehat{\mathbf{A}}, \widehat{\mathbf{C}}, \widehat{\mathbf{G}}, \widehat{\mathbf{H}}$, and $\widehat{\mathbf{K}}$. Due to the stability of $\mathbf{W}^{T} \mathbf{A V}$, the classical BTMR estimate for the error in the observations and the outputs can be shown to hold true.

Theorem 3.1. Let $\mathbf{z}(t), \mathbf{q}(t), t \in[0, T]$, and $\widehat{\mathbf{z}}(t), \widehat{\mathbf{q}}(t), t \in[0, T]$, be the observations and outputs of the full order and the reduced order optimality system as given by $(3.2 \mathrm{~b}),(3.3 \mathrm{~b})$ and $(3.7 \mathrm{~b}),(3.8 \mathrm{~b})$, and let $\sigma_{i}, 1 \leq i \leq n$, be the Hankel singular values from the singular value decomposition (3.5). Moreover, suppose that $\mathbf{v}_{H}(0)=0$ and $\boldsymbol{\lambda}_{H}(T)=0$. Then, there holds

$$
\begin{array}{r}
\|\mathbf{z}-\widehat{\mathbf{z}}\|_{L^{2}} \leq 2\|\mathbf{f}\|_{L^{2}}\left(\sigma_{p+1}+\cdots+\sigma_{n}\right), \\
\|\mathbf{q}-\widehat{\mathbf{q}}\|_{L^{2}} \leq 2\|\widehat{\mathbf{z}}\|_{L^{2}}\left(\sigma_{p+1}+\cdots+\sigma_{n}\right) .
\end{array}
$$

Proof. We refer to section 7 in [28]. 


\section{Domain decomposition and balanced truncation model reduction}

For optimal design problems associated with linear state equations, where the design only effects a relatively small part of the computational domain, the nonlinearity is thus restricted to that part and motivates to consider a combination of domain decomposition and BTMR. Let us consider a domain $\Omega(\theta)$ such that

$$
\overline{\Omega(\theta)}=\bar{\Omega}_{1} \cup \overline{\Omega_{2}(\theta)} \quad, \quad \Omega_{1} \cap \Omega_{2}(\theta)=\emptyset \quad, \quad \Gamma(\theta):=\bar{\Omega}_{1} \cap \overline{\Omega_{2}(\theta)},
$$

where the local area of interest is $\Omega_{2}(\theta)$, whereas the design variables $\theta$ do not apply to the rest $\Omega_{1}$ of the computational domain. The fine-scale model results from a spatial discretization by P2-P1 Taylor-Hood elements with respect to a simplicial triangulation of the computational domain which gives rise to a differentialalgebraic system. The fine-scale model is used only in the local area of interest, whereas a reduced order model based on balanced truncation is used for the rest of the domain. The objective functional

$$
\begin{aligned}
\mathbf{J}(\mathbf{v}, \mathbf{p}, \theta) & :=\mathbf{J}_{1}(\mathbf{v}, \mathbf{p})+\mathbf{J}_{2}(\mathbf{v}, \mathbf{p}, \theta), \\
\mathbf{J}_{1}(\mathbf{v}, \mathbf{p}) & :=\int_{0}^{T}\left|\mathbf{C}_{1} \mathbf{v}_{1}(t)+\mathbf{D}_{1} \mathbf{p}_{1}(t)+\mathbf{F}_{1} \mathbf{f}(t)-\mathbf{d}(t)\right|^{2} d t, \\
\mathbf{J}_{2}(\mathbf{v}, \mathbf{p}, \theta) & :=\int_{0}^{T} \boldsymbol{\ell}\left(\mathbf{v}_{2}(t), \mathbf{p}_{2}(t), \mathbf{v}_{\Gamma}(t), t, \theta\right) d t,
\end{aligned}
$$

is assumed to consist of an objective functional $\mathbf{J}_{1}$ of tracking type for subdomain $\Omega_{1}$, depending only on the velocity and the pressure in $\Omega_{1}$, and an objective functional $\mathbf{J}_{2}$ for subdomain $\Omega_{2}(\theta)$, depending on the velocities in $\Omega_{2}(\theta)$ and on the interface $\Gamma(\theta)$ as well as on the pressure in $\Omega_{2}(\theta)$.

Grouping the state variables according to $\mathbf{x}_{i}:=\left(\mathbf{v}_{i}, \mathbf{p}_{i}\right)^{T}, 1 \leq i \leq 2$, and $\mathbf{x}_{\Gamma}:=$ $\left(\mathbf{v}_{\Gamma}, \mathbf{p}_{0}\right)$, where the semi-discrete pressure consists of the sum of the subdomain pressures with zero average on each subdomain and constant pressures for each subdomain comprised to the constant vector $\mathbf{p}_{0}$, the semi-discretized domain decomposed Stokes system can be written in block structured form according to

$$
\begin{aligned}
& \left(\begin{array}{ccc}
\mathbf{E}_{1} & \mathbf{0} & \mathbf{0} \\
\mathbf{0} & \mathbf{E}_{2}(\theta) & \mathbf{0} \\
\mathbf{0} & \mathbf{0} & \mathbf{E}_{\Gamma}(\theta)
\end{array}\right) \frac{d}{d t}\left(\begin{array}{c}
\mathbf{x}_{1} \\
\mathbf{x}_{2} \\
\mathbf{x}_{\Gamma}
\end{array}\right)= \\
& -\left(\begin{array}{cccc}
\mathbf{S}_{11} & \mathbf{0} & \mathbf{S}_{1 \Gamma} \\
\mathbf{0} & \mathbf{S}_{22}(\theta) & \mathbf{S}_{2 \Gamma}(\theta) \\
\mathbf{S}_{1 \Gamma}^{T} & \mathbf{S}_{2 \Gamma}^{T}(\theta) & \mathbf{S}_{\Gamma \Gamma}(\theta)
\end{array}\right)\left(\begin{array}{l}
\mathbf{x}_{1} \\
\mathbf{x}_{2} \\
\mathbf{x}_{\Gamma}
\end{array}\right)+\left(\begin{array}{c}
\mathbf{H}_{1} \\
\mathbf{H}_{2}(\theta) \\
\mathbf{H}_{\Gamma}(\theta)
\end{array}\right) \mathbf{f} .
\end{aligned}
$$

Here, the singular block matrices $\mathbf{E}_{1}, \mathbf{E}_{2}(\theta)$ and $\mathbf{E}_{\Gamma}(\theta)$ are given by

$$
\mathbf{E}_{1}:=\left(\begin{array}{cc}
\mathbf{M}_{1} & \mathbf{0} \\
\mathbf{0} & \mathbf{0}
\end{array}\right), \mathbf{E}_{2}(\theta):=\left(\begin{array}{cc}
\mathbf{M}_{2}(\theta) & \mathbf{0} \\
\mathbf{0} & \mathbf{0}
\end{array}\right), \mathbf{E}_{\Gamma}(\theta):=\left(\begin{array}{cc}
\mathbf{M}_{\Gamma}(\theta) & \mathbf{0} \\
\mathbf{0} & \mathbf{0}
\end{array}\right)
$$


whereas $\mathbf{S}_{11}, \mathbf{S}_{22}(\theta)$ and $\mathbf{S}_{\Gamma \Gamma}(\theta)$ are the Stokes matrices associated with the subdomains $\Omega_{1}, \Omega_{2}(\theta)$ and the interface $\Gamma(\theta)$

$$
\begin{aligned}
\mathbf{S}_{11}:=\left(\begin{array}{cc}
\mathbf{A}_{11} & \mathbf{B}_{11}^{T} \\
\mathbf{B}_{11} & \mathbf{0}
\end{array}\right) \quad, \quad \mathbf{S}_{22}(\theta):=\left(\begin{array}{cc}
\mathbf{A}_{22}(\theta) & \mathbf{B}_{22}^{T}(\theta) \\
\mathbf{B}_{22}(\theta) & \mathbf{0}
\end{array}\right), \\
\mathbf{S}_{\Gamma \Gamma}(\theta):=\left(\begin{array}{cc}
\mathbf{A}_{\Gamma \Gamma}(\theta) & \mathbf{B}_{0}^{T} \\
\mathbf{B}_{0} & \mathbf{0}
\end{array}\right),
\end{aligned}
$$

and $\mathbf{S}_{1 \Gamma}, \mathbf{S}_{2 \Gamma}(\theta)$ are of the form

$$
\mathbf{S}_{1 \Gamma}:=\left(\begin{array}{cc}
\mathbf{A}_{11} & \mathbf{B}_{11}^{T} \\
\mathbf{B}_{11} & \mathbf{0}
\end{array}\right) \quad, \quad \mathbf{S}_{2 \Gamma}(\theta):=\left(\begin{array}{cc}
\mathbf{A}_{2 \Gamma}(\theta) & \mathbf{0} \\
\mathbf{B}_{2 \Gamma}(\theta) & \mathbf{0}
\end{array}\right) .
$$

Finally, $\mathbf{H}_{1}, \mathbf{H}_{2}(\theta)$ and $\mathbf{H}_{\Gamma}(\theta)$ are given by

$$
\mathbf{H}_{1}:=\left(\begin{array}{c}
\mathbf{K}_{1} \\
\mathbf{L}_{1}
\end{array}\right) \quad, \quad \mathbf{H}_{2}(\theta):=\left(\begin{array}{c}
\mathbf{K}_{2}(\theta) \\
\mathbf{L}_{2}(\theta)
\end{array}\right) \quad, \quad \mathbf{H}_{\Gamma}(\theta):=\left(\begin{array}{c}
\mathbf{K}_{\Gamma}(\theta) \\
\mathbf{L}_{0}(\theta)
\end{array}\right) .
$$

We now focus on the optimality system associated with subdomain $\Omega_{1}$.

Lemma 4.1. The optimality system associated with subdomain $\Omega_{1}$ consists of the state equations

$$
\begin{aligned}
& \left(\begin{array}{cc}
\mathbf{M}_{\mathbf{1}} & \mathbf{0} \\
\mathbf{0} & \mathbf{0}
\end{array}\right) \frac{d}{d t}\left(\begin{array}{l}
\mathbf{v}_{1}(t) \\
\mathbf{p}_{1}(t)
\end{array}\right)= \\
& -\left(\begin{array}{cc}
\mathbf{A}_{11} & \mathbf{B}_{11}^{T} \\
\mathbf{B}_{11} & \mathbf{0}
\end{array}\right)\left(\begin{array}{l}
\mathbf{v}_{1}(t) \\
\mathbf{p}_{1}(t)
\end{array}\right)-\left(\begin{array}{ll}
\mathbf{A}_{1 \Gamma} & \mathbf{0} \\
\mathbf{B}_{1 \Gamma} & \mathbf{0}
\end{array}\right)\left(\begin{array}{l}
\mathbf{v}_{\Gamma}(t) \\
\mathbf{p}_{0}(t)
\end{array}\right)+\left(\begin{array}{c}
\mathbf{K}_{1} \\
\mathbf{L}_{1}
\end{array}\right) \mathbf{f}(t), \\
& \mathbf{z}_{1}(t)=\mathbf{C}_{1} \mathbf{v}_{1}(t)+\mathbf{D}_{1} \mathbf{p}_{1}(t)+\mathbf{D}_{0} \mathbf{p}_{0}(t)+\mathbf{F}_{1} \mathbf{f}(t)-\mathbf{d}(t),
\end{aligned}
$$

and the adjoint state equations

$$
\begin{aligned}
& -\left(\begin{array}{cc}
\mathbf{M}_{1} & \mathbf{0} \\
\mathbf{0} & \mathbf{0}
\end{array}\right) \frac{d}{d t}\left(\begin{array}{c}
\boldsymbol{\lambda}_{1}(t) \\
\boldsymbol{\kappa}_{1}(t)
\end{array}\right)= \\
& -\left(\begin{array}{cc}
\mathbf{A}_{11} & \mathbf{B}_{11}^{T} \\
\mathbf{B}_{11} & \mathbf{0}
\end{array}\right)\left(\begin{array}{c}
\boldsymbol{\lambda}_{1}(t) \\
\boldsymbol{\kappa}_{1}(t)
\end{array}\right)-\left(\begin{array}{cc}
\mathbf{A}_{1 \Gamma} & \mathbf{0} \\
\mathbf{B}_{1 \Gamma} & \mathbf{0}
\end{array}\right)\left(\begin{array}{c}
\boldsymbol{\lambda}_{\Gamma}(t) \\
\boldsymbol{\kappa}_{0}(t)
\end{array}\right)-\left(\begin{array}{c}
\mathbf{C}_{1}^{T} \\
\mathbf{F}_{1}^{T}
\end{array}\right) \mathbf{z}_{1}(t) \\
& \mathbf{q}_{1}(t)=\mathbf{K}_{1}^{T} \boldsymbol{\lambda}_{1}(t)+\mathbf{L}_{1}^{T} \boldsymbol{\kappa}_{1}(t)+\mathbf{D}_{1}^{T} \mathbf{z}_{1}(t) .
\end{aligned}
$$

Proof. The proof follows readily from (4.2) and (4.3).

We see that (4.4a),(4.4b) and (4.5a),(4.5b) have exactly the form which we considered before in Section 3. Hence, it is directly amenable to the application of BTMR. 
Lemma 4.2. There exist projection matrices $\mathbf{V}_{1}, \mathbf{W}_{1}$ such that the reduced state equations associated with subdomain $\Omega_{1}$ are of the form

$$
\begin{gathered}
\frac{d}{d t} \widehat{\mathbf{v}}_{1}(t)=-\mathbf{W}_{1}^{T} \mathbf{A}_{11} \mathbf{V}_{1} \widehat{\mathbf{v}}_{1}(t)-\mathbf{W}_{1}^{T} \widetilde{\mathbf{B}}_{1}\left(\begin{array}{c}
\widehat{\mathbf{v}}_{\Gamma}(t) \\
\widehat{\mathbf{p}}_{0}(t) \\
\mathbf{f}(t)
\end{array}\right) \\
\left(\begin{array}{c}
\widehat{\mathbf{z}}_{v, \Gamma}(t) \\
\widehat{\mathbf{z}}_{p, \Gamma}(t) \\
\widehat{\mathbf{z}}_{1}(t)
\end{array}\right)=\widetilde{\mathbf{C}}_{1} \mathbf{V}_{1} \widehat{\mathbf{v}}_{1}(t)+\widetilde{\mathbf{D}}_{1}\left(\begin{array}{c}
\widehat{\mathbf{v}}_{\Gamma}(t) \\
\widehat{\mathbf{p}}_{0}(t) \\
\mathbf{f}(t)
\end{array}\right)-\widetilde{\mathbf{H}}_{1} \frac{d}{d t}\left(\begin{array}{c}
\widehat{\mathbf{v}}_{\Gamma}(t) \\
\widehat{\mathbf{p}}_{0}(t) \\
\mathbf{f}(t)
\end{array}\right)
\end{gathered}
$$

whereas the reduced adjoint state equations are given by

$$
\begin{gathered}
-\frac{d}{d t} \widehat{\boldsymbol{\lambda}}_{1}(t)=-\mathbf{V}_{1}^{T} \mathbf{A}_{11} \mathbf{W}_{1} \widehat{\boldsymbol{\lambda}}_{1}(t)+\mathbf{V}_{1}^{T} \widetilde{\mathbf{C}}_{1}\left(\begin{array}{c}
\widehat{\boldsymbol{\lambda}}_{1}(t) \\
\widehat{\boldsymbol{\kappa}}_{0}(t) \\
-\widehat{\mathbf{z}}_{1}(t)
\end{array}\right), \\
\left(\begin{array}{c}
\widehat{\mathbf{q}}_{v, \Gamma}(t) \\
\widehat{\mathbf{q}}_{p, \Gamma}(t) \\
\widehat{\mathbf{q}}_{1}(t)
\end{array}\right)=-\widetilde{\mathbf{B}}_{1}^{T} \mathbf{W}_{1} \widehat{\lambda}_{1}(t)+\widetilde{\mathbf{D}}_{1}^{T}\left(\begin{array}{c}
\widehat{\boldsymbol{\lambda}}_{1}(t) \\
\widehat{\boldsymbol{\kappa}}_{0}(t) \\
-\widehat{\mathbf{z}}_{1}(t)
\end{array}\right)+\widetilde{\mathbf{H}}_{1}^{T} \frac{d}{d t}\left(\begin{array}{c}
\widehat{\boldsymbol{\lambda}}_{1}(t) \\
\widehat{\boldsymbol{\kappa}}_{0}(t) \\
-\widehat{\mathbf{z}}_{1}(t)
\end{array}\right) .
\end{gathered}
$$

Proof. The construction of the projection matrices is done as described in the previous section.

Since we do not apply BTMR neither to subdomain $\Omega_{2}(\theta)$ nor to the interface $\Gamma(\theta)$, the corresponding state and adjoint state equations can be derived in a straightforward way.

Lemma 4.3. State and adjoint state equations associated with the subdomain $\Omega_{2}(\theta)$

$$
\begin{aligned}
& \left(\begin{array}{cc}
\mathbf{M}_{2}(\theta) & \mathbf{0} \\
\mathbf{0} & \mathbf{0}
\end{array}\right) \frac{d}{d t}\left(\begin{array}{c}
\widehat{\mathbf{v}}_{2}(t) \\
\widehat{\mathbf{p}}_{2}(t)
\end{array}\right)=-\left(\begin{array}{cc}
\mathbf{A}_{22}(\theta) & \mathbf{B}_{22}^{T}(\theta) \\
\mathbf{B}_{22}(\theta) & \mathbf{0}
\end{array}\right)\left(\begin{array}{l}
\widehat{\mathbf{v}}_{2}(t) \\
\widehat{\mathbf{p}}_{2}(t)
\end{array}\right) \\
& -\left(\begin{array}{ll}
\mathbf{A}_{2 \Gamma}(\theta) & \mathbf{0} \\
\mathbf{B}_{2 \Gamma}(\theta) & \mathbf{0}
\end{array}\right)\left(\begin{array}{c}
\widehat{\mathbf{v}}_{\Gamma}(t) \\
\widehat{\mathbf{p}}_{0}(t)
\end{array}\right)+\left(\begin{array}{c}
\mathbf{K}_{2}(\theta) \\
\mathbf{L}_{2}(\theta)
\end{array}\right) \mathbf{f}(t), \\
& -\left(\begin{array}{cc}
\mathbf{M}_{2}(\theta) & \mathbf{0} \\
\mathbf{0} & \mathbf{0}
\end{array}\right) \frac{d}{d t}\left(\begin{array}{c}
\widehat{\boldsymbol{\lambda}}_{2}(t) \\
\widehat{\boldsymbol{\kappa}}_{2}(t)
\end{array}\right)=-\left(\begin{array}{cc}
\mathbf{A}_{22}(\theta) & \mathbf{B}_{22}^{T}(\theta) \\
\mathbf{B}_{22}(\theta) & \mathbf{0}
\end{array}\right)\left(\begin{array}{l}
\widehat{\boldsymbol{\lambda}}_{2}(t) \\
\widehat{\boldsymbol{\kappa}}_{2}(t)
\end{array}\right) \\
& -\left(\begin{array}{ll}
\mathbf{A}_{2 \Gamma}(\theta) & \mathbf{0} \\
\mathbf{B}_{2 \Gamma}(\theta) & \mathbf{0}
\end{array}\right)\left(\begin{array}{c}
\widehat{\boldsymbol{\lambda}}_{\Gamma}(t) \\
\widehat{\boldsymbol{\kappa}}_{0}(t)
\end{array}\right)-\left(\begin{array}{c}
\nabla_{\widehat{v}_{2}} \ell\left(\widehat{\mathbf{v}}_{2}, \widehat{\mathbf{p}}_{2}, \widehat{\mathbf{v}}_{\Gamma}, \widehat{\mathbf{p}}_{0}, t, \theta\right) \\
\nabla_{\widehat{\mathbf{p}}_{2}} \boldsymbol{\ell}\left(\widehat{\mathbf{v}}_{2}, \widehat{\mathbf{p}}_{2}, \widehat{\mathbf{v}}_{\Gamma}, \widehat{\mathbf{p}}_{0}, t, \theta\right)
\end{array}\right) .
\end{aligned}
$$


State and adjoint state equations associated with the interface $\Gamma(\theta)$

$$
\begin{aligned}
& \left(\begin{array}{cc}
\mathbf{M}_{\Gamma}(\theta) & \mathbf{0} \\
\mathbf{0} & \mathbf{0}
\end{array}\right) \frac{d}{d t}\left(\begin{array}{c}
\widehat{\mathbf{v}}_{\Gamma}(t) \\
\widehat{\mathbf{p}}_{0}(t)
\end{array}\right)=-\left(\begin{array}{cc}
\mathbf{A}_{\Gamma \Gamma}(\theta) & \mathbf{B}_{0}^{T}(\theta) \\
\mathbf{B}_{0}(\theta) & \mathbf{0}
\end{array}\right)\left(\begin{array}{l}
\widehat{\mathbf{v}}_{\Gamma}(t) \\
\widehat{\mathbf{p}}_{0}(t)
\end{array}\right) \\
& +\left(\begin{array}{c}
\widehat{\mathbf{z}}_{v, \Gamma}(t) \\
\widehat{\mathbf{z}}_{p, \Gamma}(t)
\end{array}\right)-\left(\begin{array}{cc}
\mathbf{A}_{2 \Gamma}^{T}(\theta) & \mathbf{B}_{2 \Gamma}^{T}(\theta) \\
\mathbf{0} & \mathbf{0}
\end{array}\right)\left(\begin{array}{c}
\widehat{\mathbf{v}}_{2}(t) \\
\widehat{\mathbf{p}}_{2}(t)
\end{array}\right)+\left(\begin{array}{c}
\mathbf{K}_{\Gamma}(\theta) \\
\mathbf{L}_{0}(\theta)
\end{array}\right) \mathbf{f}(t), \\
& -\left(\begin{array}{cc}
\mathbf{M}_{\Gamma}(\theta) & \mathbf{0} \\
\mathbf{0} & \mathbf{0}
\end{array}\right) \frac{d}{d t}\left(\begin{array}{c}
\widehat{\boldsymbol{\lambda}}_{\Gamma}(t) \\
\widehat{\boldsymbol{\kappa}}_{0}(t)
\end{array}\right)=-\left(\begin{array}{cc}
\mathbf{A}_{\Gamma \Gamma}(\theta) & \mathbf{B}_{0}^{T}(\theta) \\
\mathbf{B}_{0}(\theta) & \mathbf{0}
\end{array}\right)\left(\begin{array}{l}
\widehat{\boldsymbol{\lambda}}_{\Gamma}(t) \\
\widehat{\boldsymbol{\kappa}}_{0}(t)
\end{array}\right) \\
& +\left(\begin{array}{c}
\widehat{\mathbf{q}}_{v, \Gamma}(t) \\
\widehat{\mathbf{q}}_{p, \Gamma}(t)
\end{array}\right)-\left(\begin{array}{cc}
\mathbf{A}_{2 \Gamma}^{T}(\theta) & \mathbf{B}_{2 \Gamma}^{T}(\theta) \\
\mathbf{0} & \mathbf{0}
\end{array}\right)\left(\begin{array}{c}
\widehat{\boldsymbol{\lambda}}_{2}(t) \\
\widehat{\boldsymbol{\kappa}}_{2}(t)
\end{array}\right)-\left(\begin{array}{c}
\nabla_{\mathbf{v}_{\Gamma}} \boldsymbol{\ell}(\cdots) \\
\nabla_{\mathbf{p}_{0}} \boldsymbol{\ell}(\cdots)
\end{array}\right) .
\end{aligned}
$$

The equations (4.8a),(4.8b) and (4.9a),(4.9b) are complemented by the variational inequality

$$
\begin{aligned}
& \int_{0}^{T} \nabla_{\theta} \ell\left(\mathbf{v}_{2}, \mathbf{p}_{2}, \mathbf{v}_{\Gamma}, \mathbf{p}_{0}, t, \theta\right) d t+ \\
& \int_{0}^{T}\left(\begin{array}{c}
\widehat{\boldsymbol{\mu}}_{2}(t) \\
\widehat{\boldsymbol{\mu}}_{\Gamma}(t)
\end{array}\right)^{T}\left(\begin{array}{c}
\left(\mathbf{D}_{\theta} \widehat{\mathbf{P}}_{2}(\theta)(\tilde{\theta}-\theta) \widehat{\mathbf{x}}_{2}(t)-\left(\mathbf{D}_{\theta} \widehat{\mathbf{N}}_{2}(\theta)(\tilde{\theta}-\theta) \mathbf{f}(t)\right.\right. \\
\left(\mathbf{D}_{\theta} \widehat{\mathbf{P}}_{\Gamma}(\theta)(\tilde{\theta}-\theta) \widehat{\mathbf{x}}_{\Gamma}(t)-\left(\mathbf{D}_{\theta} \widehat{\mathbf{N}}_{\Gamma}(\theta)(\tilde{\theta}-\theta) \mathbf{f}(t)\right.\right.
\end{array}\right) d t \geq 0,
\end{aligned}
$$

which is supposed to hold true for all $\tilde{\theta} \in \Theta$. Here, $\widehat{\mathbf{N}}_{2}(\theta), \widehat{\mathbf{N}}_{\Gamma}(\theta)$ and $\widehat{\mathbf{P}}_{2}(\theta), \widehat{\mathbf{P}}_{\Gamma}(\theta)$ are given by

$$
\begin{array}{r}
\widehat{\mathbf{N}}_{2}(\theta):=\left(\widehat{\mathbf{K}}_{2}(\theta), \widehat{\mathbf{L}}_{2}(\theta)\right)^{T} \quad, \quad \widehat{\mathbf{N}}_{\Gamma}(\theta):=\left(\widehat{\mathbf{K}}_{\Gamma}(\theta), \widehat{\mathbf{L}}_{0}(\theta)\right)^{T}, \\
\widehat{\mathbf{P}}_{2}(\theta) \widehat{\mathbf{x}}_{2}(t):=\widehat{\mathbf{E}}_{2}(\theta) \frac{d}{d t} \mathbf{x}_{2}(t)+\widehat{\mathbf{S}}_{22}(\theta) \widehat{\mathbf{x}}_{2}(t), \\
\widehat{\mathbf{P}}_{\Gamma}(\theta) \widehat{\mathbf{x}}_{\Gamma}(t):=\widehat{\mathbf{E}}_{\Gamma}(\theta) \frac{d}{d t} \widehat{\mathbf{x}}_{\Gamma}(t)+\widehat{\mathbf{S}}_{\Gamma \Gamma}(\theta) \widehat{\mathbf{x}}_{\Gamma}(t) .
\end{array}
$$

The following result can be verified by straightforward computation.

Theorem 4.4. The reduced order optimality system (4.6a)-(4.10) represents the first order necessary optimality conditions for the reduced order optimization problem

$$
\inf _{\theta \in \Theta} \widehat{\mathbf{J}}(\theta) \quad, \quad \widehat{\mathbf{J}}(\theta):=\widehat{\mathbf{J}}_{1}\left(\widehat{\mathbf{v}}_{1}, \widehat{\mathbf{p}}_{1}, \widehat{\mathbf{v}}_{\Gamma}, \widehat{\mathbf{p}}_{0}\right)+\widehat{\mathbf{J}}_{2}\left(\widehat{\mathbf{v}}_{2}, \widehat{\mathbf{p}}_{2}, \widehat{\mathbf{v}}_{\Gamma}, \widehat{\mathbf{p}}_{0}, \theta\right)
$$

where the the reduced order functionals $\widehat{\mathbf{J}}_{1}$ and $\widehat{\mathbf{J}}_{2}$ are given by

$$
\begin{aligned}
& \widehat{\mathbf{J}}_{1}\left(\widehat{\mathbf{v}}_{1}, \widehat{\mathbf{p}}_{1}, \widehat{\mathbf{v}}_{\Gamma}, \widehat{\mathbf{p}}_{0}\right):=\frac{1}{2} \int_{0}^{T}\left|\widehat{\mathbf{z}}_{1}\right|^{2} d t, \\
& \widehat{\mathbf{J}}_{2}\left(\widehat{\mathbf{v}}_{2}, \widehat{\mathbf{p}}_{2}, \widehat{\mathbf{v}}_{\Gamma}, \widehat{\mathbf{p}}_{0}, \theta\right):=\int_{0}^{T} \boldsymbol{\ell}\left(\widehat{\mathbf{v}}_{2}, \widehat{\mathbf{p}}_{2}, \widehat{\mathbf{v}}_{\Gamma}, \widehat{\mathbf{p}}_{0}, t, \theta\right) d t .
\end{aligned}
$$




\section{A priori estimates of the modeling error}

We now focus our attention on an a priori analysis of the modeling error due to the approximation of the full order model by the reduced order model obtained by the application of the combined domain decomposition and balanced truncation model reduction approach. We will show that under some assumptions the error in the optimal design can be bounded from above by the sum of the remaining Hankel singular values, i.e., we are able to derive an upper bound of the same form as in the standard BTMR estimates:

$$
\left\|\theta^{*}-\widehat{\theta}^{*}\right\| \leq C\left(\sigma_{p+1}+\cdots+\sigma_{n}\right) .
$$

One of these assumptions is to require the objective functional $\mathbf{J}$ to be strongly convex.

$\left(\mathbf{A}_{1}\right)$ There exists a constant $\kappa>0$ such that for all $\widehat{\theta}, \theta \in \Theta$ there holds

$$
(\nabla \mathbf{J}(\widehat{\theta})-\nabla \mathbf{J}(\theta))^{T}(\widehat{\theta}-\theta) \geq \kappa\|\widehat{\theta}-\theta\|^{2}
$$

Then, it is easy to see that the error in the optimal design is bounded from above by the difference of the gradients of the objective functional for the reduced optimization problem and the gradient of objective functional for the full optimization problem at optimality $\widehat{\theta}^{*}$.

Lemma 5.1. Assume that the objective functional $J$ satisfies $\left(\mathbf{A}_{\mathbf{1}}\right)$. Then, if $\theta^{*} \in \Theta$ and $\widehat{\theta}^{*} \in \Theta$ are the solutions of the full order and the reduced order optimization problem, there holds

$$
\left\|\theta^{*}-\widehat{\theta}^{*}\right\| \leq \kappa^{-1}\left\|\nabla \widehat{\mathbf{J}}\left(\widehat{\theta}^{*}\right)-\nabla \mathbf{J}\left(\widehat{\theta}^{*}\right)\right\| .
$$

Proof. Obviously, we have

$$
\left.\begin{array}{l}
\nabla \mathbf{J}\left(\theta^{*}\right)^{T}\left(\theta-\theta^{*}\right) \geq 0 \\
\nabla \widehat{\mathbf{J}}\left(\widehat{\theta}^{*}\right)^{T}\left(\theta-\widehat{\theta}^{*}\right) \geq 0
\end{array}\right\} \quad \Longrightarrow \quad\left(\nabla \mathbf{J}\left(\theta^{*}\right)-\nabla \widehat{\mathbf{J}}\left(\widehat{\theta}^{*}\right)\right)^{T}\left(\widehat{\theta}^{*}-\theta^{*}\right) \geq 0
$$

Combining (5.2) with the strong convexity of $\mathbf{J}$ allows to deduce (5.1).

Lemma 5.1 tells us that we have to provide an upper bound for the difference of these gradients. To this end, we further have to require that the objective functional $\mathbf{J}_{1}$ associated with the subdomain $\Omega_{1}$ does not explicitly depend on the pressure. Moreover, as far as the objective functional $\mathbf{J}_{2}$ associated with the subdomain $\Omega_{2}(\theta)$ is concerned, we assume that the derivatives with respect to the state variables $\mathbf{v}_{2}, \mathbf{v}_{\Gamma}, \mathbf{p}_{2}$ and $\mathbf{p}_{0}$ are Lipschitz continuous uniformly in the design variable $\theta$.

$\left(\mathbf{A}_{2}\right)$ The objective functional $\mathbf{J}_{1}$ does not explicitly depend on the pressure, i.e., it is supposed to be of the form

$$
\mathbf{J}_{1}\left(\mathbf{v}_{1}\right)=\frac{1}{2} \int_{0}^{T}\left|\mathbf{C}_{1} \mathbf{v}(t)+\mathbf{F}_{1} \mathbf{f}(t)-\mathbf{d}(t)\right|^{2} d t .
$$


$\left(\mathbf{A}_{\mathbf{3}}\right)$ The integrand $\boldsymbol{\ell}$ in the objective functional

$$
\mathbf{J}_{2}\left(\mathbf{x}_{2}, \mathbf{x}_{\Gamma}, \theta\right)=\frac{1}{2} \int_{0}^{T} \ell\left(\mathbf{x}_{2}, \mathbf{x}_{\Gamma}, t, \theta\right) d t
$$

where $\mathbf{x}_{2}:=\left(\mathbf{v}_{2}, \mathbf{p}_{2}\right)^{T}, \mathbf{x}_{\Gamma}:=\left(\mathbf{v}_{\Gamma}, \mathbf{p}_{0}\right)^{T}$, satisfies the Lipschitz conditions

$$
\left\|\nabla_{\mathbf{w}} \ell\left(\mathbf{x}_{2}, \mathbf{x}_{\Gamma}, t, \theta\right)-\nabla_{\mathbf{w}} \ell\left(\mathbf{x}_{2}^{\prime}, \mathbf{x}_{\Gamma}^{\prime}, t, \theta\right)\right\| \leq L_{1}\left(\left\|\mathbf{x}_{2}-\mathbf{x}_{2}^{\prime}\right\|^{2}+\left\|\mathbf{x}_{\Gamma}-\mathbf{x}_{\Gamma}^{\prime}\right\|^{2}\right)^{1 / 2}
$$

uniformly in $\theta \in \Theta$ and $t \in[0, T]$, where $\mathbf{w} \in\left\{\mathbf{v}_{2}, \mathbf{v}_{\Gamma}, \mathbf{p}_{2}, \mathbf{p}_{0}\right\}$.

Under these two requirements and the additional assumption that the Jacobians of the $\theta$-dependent system matrices are uniformly bounded in $\theta$, it follows that the difference in the gradients can be bounded from above by the $L^{2}$-norms of the differences between the full and the reduced states as well as the full and reduced adjoint states.

Lemma 5.2. Assume that $\left(\mathbf{A}_{\mathbf{2}}\right),\left(\mathbf{A}_{\mathbf{3}}\right)$ hold true and suppose that the Jacobians of the matrices $\mathbf{M}_{2}(\theta), \mathbf{M}_{\Gamma}(\theta)$ etc. are uniformly bounded in $\theta$. Then, there exists a constant $C>0$ such that for $\theta \in \Theta$

$$
\|\nabla \mathbf{J}(\theta)-\nabla \widehat{\mathbf{J}}(\theta)\| \leq C\left(\left\|\left(\begin{array}{c}
\mathbf{x}_{2}-\widehat{\mathbf{x}}_{2} \\
\mathbf{x}_{\Gamma}-\widehat{\mathbf{x}}_{\Gamma}
\end{array}\right)\right\|_{L^{2}}+\left\|\left(\begin{array}{c}
\boldsymbol{\mu}_{2}-\widehat{\boldsymbol{\mu}}_{2} \\
\boldsymbol{\mu}_{\Gamma}-\widehat{\boldsymbol{\mu}}_{\Gamma}
\end{array}\right)\right\|_{L^{2}}\right)
$$

where $\mathbf{x}_{2}-\widehat{\mathbf{x}}_{2}, \mathbf{x}_{\Gamma}-\widehat{\mathbf{x}}_{\Gamma}$ and $\boldsymbol{\mu}_{2}-\widehat{\boldsymbol{\mu}}_{2}, \boldsymbol{\mu}_{\Gamma}-\widehat{\boldsymbol{\mu}}_{\Gamma}$ are given by

$$
\begin{array}{ll}
\mathbf{x}_{2}-\widehat{\mathbf{x}}_{2}=\left(\begin{array}{c}
\mathbf{v}_{2}-\widehat{\mathbf{v}}_{2} \\
\mathbf{p}_{2}-\widehat{\mathbf{p}}_{2}
\end{array}\right) \quad, \quad \mathbf{x}_{\Gamma}-\widehat{\mathbf{x}}_{\Gamma}=\left(\begin{array}{c}
\mathbf{v}_{\Gamma}-\widehat{\mathbf{v}}_{\Gamma} \\
\mathbf{p}_{0}-\widehat{\mathbf{p}}_{0}
\end{array}\right), \\
\boldsymbol{\mu}_{2}-\widehat{\boldsymbol{\mu}}_{2}=\left(\begin{array}{c}
\boldsymbol{\lambda}_{2}-\widehat{\boldsymbol{\lambda}}_{2} \\
\boldsymbol{\kappa}_{2}-\widehat{\boldsymbol{\kappa}}_{2}
\end{array}\right) \quad, \quad \boldsymbol{\mu}_{\Gamma}-\widehat{\boldsymbol{\mu}}_{\Gamma}=\left(\begin{array}{c}
\boldsymbol{\lambda}_{\Gamma}-\widehat{\boldsymbol{\lambda}}_{\Gamma} \\
\boldsymbol{\kappa}_{\mathbf{0}}-\widehat{\boldsymbol{\kappa}}_{\mathbf{0}}
\end{array}\right) .
\end{array}
$$

Proof. For $\tilde{\theta} \in \Theta$ there holds

$$
\begin{aligned}
& \nabla \mathbf{J}(\theta)^{T} \tilde{\theta}=\int_{0}^{T}\left(\nabla_{\theta} \boldsymbol{\ell}\left(\mathbf{x}_{2}, \mathbf{x}_{\Gamma}, t, \theta\right)\right)^{T} \tilde{\theta} d t+ \\
& \int_{0}^{T}\left(\begin{array}{c}
\boldsymbol{\mu}_{2}(t) \\
\boldsymbol{\lambda}_{\Gamma}(t)
\end{array}\right)^{T}\left(\begin{array}{c}
\left(\mathbf{D}_{\theta} \mathbf{P}_{2}(\theta) \tilde{\theta}\right) \mathbf{x}_{2}(t)-\left(\mathbf{D}_{\theta} \mathbf{N}_{2}(\theta) \tilde{\theta}\right) \mathbf{f}(t) \\
\left(\mathbf{D}_{\theta} \mathbf{P}_{\Gamma}(\theta) \tilde{\theta}\right) \mathbf{x}_{\Gamma}(t)-\left(\mathbf{D}_{\theta} \mathbf{N}_{\Gamma}(\theta) \tilde{\theta}\right) \mathbf{f}(t)
\end{array}\right) d t .
\end{aligned}
$$

Likewise, we have

$$
\begin{aligned}
& \nabla \widehat{\mathbf{J}}(\theta)^{T} \tilde{\theta}=\int_{0}^{T}\left(\nabla_{\theta} \boldsymbol{\ell}\left(\widehat{\mathbf{x}}_{2}, \widehat{\mathbf{x}}_{\Gamma}, t, \theta\right)\right)^{T} \tilde{\theta} d t+ \\
& \int_{0}^{T}\left(\begin{array}{c}
\widehat{\boldsymbol{\mu}}_{2}(t) \\
\widehat{\boldsymbol{\lambda}}_{\Gamma}(t)
\end{array}\right)^{T}\left(\begin{array}{c}
\left(\mathbf{D}_{\theta} \mathbf{P}_{2}(\theta) \tilde{\theta}\right) \widehat{\mathbf{x}}_{2}(t)-\left(\mathbf{D}_{\theta} \mathbf{N}_{2}(\theta) \tilde{\theta}\right) \mathbf{f}(t) \\
\left(\mathbf{D}_{\theta} \mathbf{P}_{\Gamma}(\theta) \tilde{\theta}\right) \widehat{\mathbf{x}}_{\Gamma}(t)-\left(\mathbf{D}_{\theta} \mathbf{N}_{\Gamma}(\theta) \tilde{\theta}\right) \mathbf{f}(t)
\end{array}\right) d t .
\end{aligned}
$$


Subtracting (5.5) from (5.4) and using $\left(\mathbf{A}_{\mathbf{3}}\right)$ gives the assertion.

Consequently, it remains to estimate the modeling error in those states and adjoint states associated with subdomain $\Omega_{2}(\theta)$ and the interface $\Gamma(\theta)$. For this purpose, we suppose stability of the semi-discrete Stokes system and the subsystem associated with subdomain $\Omega_{1}$.

$\left(\mathbf{A}_{4}\right)$ The matrix $\mathbf{A}(\theta) \in \mathbb{R}^{n \times n}$ is symmetric positive definite and the matrix $\mathbf{B}(\theta) \in \mathbb{R}^{m \times n}$ has rank $m$. The generalized eigenvalues of $(\mathbf{A}(\theta), \mathbf{M}(\theta))$ have positive real part.

The matrix $\mathbf{A}_{11}(\theta) \in \mathbb{R}^{n_{1} \times n_{1}}$ is symmetric positive definite and the ma$\operatorname{trix} \mathbf{B}_{11}(\theta) \in \mathbb{R}^{m_{1} \times n_{1}}$ has rank $m_{1}$. The generalized eigenvalues of $\left(\mathbf{A}_{11}(\theta)\right.$, $\left.\mathbf{M}_{11}(\theta)\right)$ have positive real part.

For the modeling errors in the velocities and the pressures we will show that they can be bounded from above by the sum of the remaining Hankel singular values. The same holds true for the errors in the observations in $\Omega_{1}$ and on the interface.

Lemma 5.3. Let $\mathbf{x}=\left(\mathbf{x}_{1}, \mathbf{x}_{2}, \mathbf{x}_{\Gamma}\right)^{T}$ with $\mathbf{x}_{i}=\left(\mathbf{v}_{i}, \mathbf{p}_{i}\right)^{T}, 1 \leq i \leq 2, \mathbf{x}_{\Gamma}=\left(\mathbf{v}_{\Gamma}, \mathbf{p}_{\Gamma}\right)^{T}$ and $\widehat{\mathbf{x}}=\left(\widehat{\mathbf{x}}_{1}, \widehat{\mathbf{x}}_{2}, \widehat{\mathbf{x}}_{\Gamma}\right)^{T}$ with $\widehat{\mathbf{x}}_{1}=\widehat{\mathbf{v}}_{1}, \widehat{\mathbf{x}}_{2}=\left(\widehat{\mathbf{v}}_{2}, \widehat{\mathbf{p}}_{2}\right)^{T}, \widehat{\mathbf{x}}_{\Gamma}=\left(\widehat{\mathbf{v}}_{\Gamma}, \widehat{\mathbf{p}}_{0}\right)^{T}$, be the states satisfying the optimality systems associated with the full order and the reduced order model. Then, under assumption $\left(\mathbf{A}_{\mathbf{4}}\right)$ and for $\mathbf{v}_{1}^{(0)}=0$ there exists $C>0$ such that

$$
\begin{array}{r}
\left\|\left(\begin{array}{c}
\mathbf{v}_{2}-\widehat{\mathbf{v}}_{2} \\
\mathbf{v}_{\Gamma}-\widehat{\mathbf{v}}_{\Gamma}
\end{array}\right)\right\|_{L^{2}} \leq C\left\|\left(\begin{array}{c}
\mathbf{f} \\
\widehat{\mathbf{x}}_{\Gamma}
\end{array}\right)\right\|_{L^{2}}\left(\sigma_{p+1}+\cdots+\sigma_{n}\right), \\
\left\|\left(\begin{array}{c}
\mathbf{p}_{2}-\widehat{\mathbf{p}}_{2} \\
\mathbf{p}_{0}-\widehat{\mathbf{p}}_{0}
\end{array}\right)\right\|_{L^{2}} \leq C\left\|\left(\begin{array}{c}
\mathbf{f} \\
\widehat{\mathbf{x}}_{\Gamma}
\end{array}\right)\right\|_{L^{2}}\left(\sigma_{p+1}+\cdots+\sigma_{n}\right), \\
\left\|\left(\begin{array}{c}
\mathbf{z}_{1}-\widehat{\mathbf{z}}_{1} \\
\mathbf{z}_{v, \Gamma}-\widehat{\mathbf{z}}_{v, \Gamma} \\
\mathbf{z}_{p, \Gamma}-\widehat{\mathbf{z}}_{p, \Gamma}
\end{array}\right)\right\|_{L^{2}} \leq C\left\|\left(\begin{array}{c}
\mathbf{f} \\
\widehat{\mathbf{x}}_{\Gamma}
\end{array}\right)\right\|_{L^{2}}\left(\sigma_{p+1}+\cdots+\sigma_{n}\right) .
\end{array}
$$

Proof. Since the full order optimality equations for subdomain $\Omega_{1}$ differ from the reduced order optimality system by the inputs from the interface $\Gamma$, we construct an auxiliary system with velocity $\widetilde{\mathbf{v}}_{1}$, pressure $\widetilde{\mathbf{p}}_{1}$, and observations $\widetilde{\mathbf{z}}$ that has the same inputs as the reduced order system.

$$
\begin{aligned}
\mathbf{E}_{1} \frac{d}{d t}\left(\begin{array}{c}
\tilde{\mathbf{v}}_{1}(t) \\
\tilde{\mathbf{p}}_{1}(t)
\end{array}\right) & =-\mathbf{S}_{1}\left(\begin{array}{c}
\tilde{\mathbf{v}}_{1}(t) \\
\tilde{\mathbf{p}}_{1}(t)
\end{array}\right)-\mathbf{S}_{1, \Gamma}\left(\begin{array}{c}
\widehat{\mathbf{v}}_{\Gamma}(t) \\
\widehat{\mathbf{p}}_{0}(t)
\end{array}\right)+\left(\begin{array}{c}
\mathbf{K}_{1} \\
\mathbf{L}_{1}
\end{array}\right) \mathbf{f}(t), \\
\tilde{\mathbf{z}}_{1}(t) & =\mathbf{C}_{1} \tilde{\mathbf{v}}_{1}(t)+\mathbf{F}_{1} \tilde{\mathbf{p}}_{1}(t)+\mathbf{F}_{0} \widehat{\mathbf{p}}_{0}(t)+\mathbf{D}_{1} \mathbf{f}(t)-\mathbf{d}(t), \\
\left(\begin{array}{c}
\tilde{\mathbf{z}}_{v, \Gamma}(t) \\
\tilde{\mathbf{z}}_{p, \Gamma}(t)
\end{array}\right) & =-\mathbf{S}_{1 \Gamma}^{T}\left(\begin{array}{c}
\tilde{\mathbf{v}}_{1}(t) \\
\tilde{\mathbf{p}}_{1}(t)
\end{array}\right), \\
\mathbf{M}_{1} \tilde{\mathbf{v}}_{1}(0) & =\mathbf{v}_{1}^{(0)}, \quad \mathbf{L}_{1} \mathbf{f}(0)=\mathbf{B}_{11} \mathbf{M}_{1}^{-1} \mathbf{v}_{1}^{(0)}+\mathbf{B}_{1 \Gamma} \mathbf{M}_{\Gamma}(\theta)^{-1} \mathbf{v}_{\Gamma}^{(0)}(\theta) .
\end{aligned}
$$

Then, the standard BT error bound tells us that the error in the observations between the auxiliary and the reduced order system can be bounded from above 
by the sum of the remaining Hankel singular values.

$$
\left\|\left(\begin{array}{c}
\tilde{\mathbf{z}}_{1}-\widehat{\mathbf{z}}_{1} \\
\tilde{\mathbf{z}}_{v, \Gamma}-\widehat{\mathbf{z}}_{v, \Gamma} \\
\tilde{\mathbf{z}}_{p, \Gamma}-\widehat{\mathbf{z}}_{p, \Gamma}
\end{array}\right)\right\|_{L^{2}} \leq 2\left(\sigma_{p+1}+\cdots+\sigma_{n}\right)\left\|\left(\begin{array}{c}
\mathbf{f} \\
\widehat{\mathbf{v}}_{\Gamma} \\
\widehat{\mathbf{p}}_{0}
\end{array}\right)\right\|_{L^{2}} .
$$

Considering the errors in the states with $\widehat{\mathbf{v}}_{1}$ and $\widehat{\mathbf{p}}_{1}$ replaced with the velocity $\widetilde{\mathbf{v}}_{1}$ and the pressure $\widetilde{\mathbf{p}}_{1}$ from the auxiliary system

$$
\mathbf{e}_{v}:=\left(\mathbf{v}_{1}-\tilde{\mathbf{v}}_{1}, \mathbf{v}_{2}-\widehat{\mathbf{v}}_{2}, \mathbf{v}_{\Gamma}-\widehat{\mathbf{v}}_{\Gamma}\right)^{T} \quad, \quad \mathbf{e}_{p}:=\left(\mathbf{p}_{1}-\tilde{\mathbf{p}}_{1}, \mathbf{p}_{2}-\widehat{\mathbf{p}}_{2}, \mathbf{p}_{0}-\widehat{\mathbf{p}}_{0}\right)^{T},
$$

we see that the errors satisfy an index 2 differential algebraic equation with the forcing term being the difference in the observations at the interface.

$$
\begin{aligned}
\mathbf{E}(\theta) \frac{d}{d t}\left(\begin{array}{c}
\mathbf{e}_{v}(t) \\
\mathbf{e}_{p}(t)
\end{array}\right) & =-\mathbf{S}(\theta)\left(\begin{array}{c}
\mathbf{e}_{v}(t) \\
\mathbf{e}_{p}(t)
\end{array}\right)+\left(\begin{array}{c}
\mathbf{g}_{1}(t) \\
\mathbf{0}
\end{array}\right), \quad t \in(0, T], \\
\mathbf{M}(\theta) \mathbf{e}_{v}(0) & =\mathbf{0} .
\end{aligned}
$$

Here, $\mathbf{g}_{1}(t):=\left(\mathbf{0}, \mathbf{0}, \tilde{\mathbf{z}}_{\mathbf{v}, \boldsymbol{\Gamma}}-\widehat{\mathbf{z}}_{\mathbf{v}, \boldsymbol{\Gamma}}\right)^{\mathbf{T}}$. Theorem 2.1 implies

$$
\begin{aligned}
& \left\|\left(\begin{array}{c}
\mathbf{v}_{1}-\tilde{\mathbf{v}}_{1} \\
\mathbf{v}_{2}-\widehat{\mathbf{v}}_{2} \\
\mathbf{v}_{\Gamma}-\widehat{\mathbf{v}}_{\Gamma}
\end{array}\right)\right\|_{L^{2}} \leq C\left\|\tilde{\mathbf{z}}_{v, \Gamma}-\widehat{\mathbf{z}}_{v, \Gamma}\right\|_{L^{2}}, \\
& \left\|\left(\begin{array}{c}
\mathbf{p}_{1}-\tilde{\mathbf{p}}_{1} \\
\mathbf{p}_{2}-\widehat{\mathbf{p}}_{2} \\
\mathbf{p}_{0}-\widehat{\mathbf{p}}_{0}
\end{array}\right)\right\|_{L^{2}} \leq C\left\|\tilde{\mathbf{z}}_{v, \Gamma}-\widehat{\mathbf{z}}_{v, \Gamma}\right\|_{L^{2}} .
\end{aligned}
$$

Using (5.7) in (5.8a) and (5.8b) results in (5.6a) and (5.6b).

For the error in the adjoint states, under assumptions $\left(\mathbf{A}_{\mathbf{2}}\right),\left(\mathbf{A}_{\mathbf{3}}\right)$, and $\left(\mathbf{A}_{\mathbf{4}}\right)$, we can derive a similar upper bound.

Lemma 5.4. Let $\mathbf{x}, \mathbf{x}_{\Gamma}$ as in Lemma 5.3 and assume that $\boldsymbol{\mu}:=\left(\boldsymbol{\mu}_{1}, \boldsymbol{\mu}_{2}, \boldsymbol{\mu}_{\Gamma}\right)^{T}$ with $\boldsymbol{\mu}_{i}:=\left(\boldsymbol{\lambda}_{i}, \boldsymbol{\kappa}_{i}\right)^{T}, 1 \leq i \leq 2, \boldsymbol{\mu}_{\Gamma}:=\left(\boldsymbol{\lambda}_{\Gamma}, \boldsymbol{\kappa}_{0}\right)^{T}$ and $\widehat{\boldsymbol{\mu}}:=\left(\widehat{\boldsymbol{\mu}}_{1}, \widehat{\boldsymbol{\mu}}_{2}, \widehat{\boldsymbol{\mu}}_{\Gamma}\right)^{T}$ with $\widehat{\boldsymbol{\mu}}_{1}:=$ $\widehat{\boldsymbol{\lambda}}_{1}, \widehat{\boldsymbol{\mu}}_{2}:=\left(\widehat{\boldsymbol{\lambda}}_{2}, \widehat{\boldsymbol{\kappa}}_{2}\right)^{T}, \widehat{\boldsymbol{\mu}}_{\Gamma}:=\left(\widehat{\boldsymbol{\lambda}}_{\Gamma}, \widehat{\boldsymbol{\kappa}}_{0}\right)^{T}$ satisfy the optimality systems associated with the full order and the reduced order model. Then, under assumptions $\left(\mathbf{A}_{\mathbf{2}}\right),\left(\mathbf{A}_{\mathbf{3}}\right)$ and for $\boldsymbol{\lambda}_{1}^{(T)}=0$ there exists $C>0$ such that

$$
\begin{gathered}
\left\|\left(\begin{array}{c}
\boldsymbol{\lambda}_{2}-\widehat{\boldsymbol{\lambda}}_{2} \\
\boldsymbol{\lambda}_{\Gamma}-\widehat{\boldsymbol{\lambda}}_{\Gamma}
\end{array}\right)\right\|_{L^{2}} \leq C\left(\left\|\left(\begin{array}{c}
\mathbf{f} \\
\widehat{\mathbf{x}}_{\Gamma}
\end{array}\right)\right\|_{L^{2}}+\left\|\left(\begin{array}{c}
\widehat{\mathbf{z}}_{1} \\
\widehat{\boldsymbol{\mu}}_{\Gamma}
\end{array}\right)\right\|_{L^{2}}\right)\left(\sigma_{p+1}+\cdot+\sigma_{n}\right), \\
\left\|\left(\begin{array}{c}
\boldsymbol{\kappa}_{2}-\widehat{\boldsymbol{\kappa}}_{2} \\
\boldsymbol{\kappa}_{0}-\widehat{\boldsymbol{\kappa}}_{0}
\end{array}\right)\right\|_{L^{2}} \leq C\left(\left\|\left(\begin{array}{c}
\mathbf{f} \\
\widehat{\mathbf{x}}_{\Gamma}
\end{array}\right)\right\|_{L^{2}}+\left\|\left(\begin{array}{c}
\widehat{\mathbf{z}}_{1} \\
\widehat{\boldsymbol{\mu}}_{\Gamma}
\end{array}\right)\right\|_{L^{2}}\right)\left(\sigma_{p+1}+\cdot+\sigma_{n}\right) .
\end{gathered}
$$

Proof. Using the same reasoning as in the proof of Lemma 5.3, we construct an appropriate auxiliary system for subdomain $\Omega_{1}$ which has the same inputs on the interface as the reduced order system in the adjoint states. For details we refer to Lemma 6.2 in [4].

Combining the results of the the previous lemmas, we obtain the desired a priori estimate of the modeling error. 
Theorem 5.5. Under assumptions $\left(A_{1}\right)-\left(A_{4}\right)$ let $\theta^{*}$ and $\widehat{\theta}^{*}$ be the optimal designs obtained by the solution of the full order and the reduced order optimization problem. Then, there exists $C>0$ such that

$$
\left\|\theta^{*}-\widehat{\theta}^{*}\right\| \leq C\left(\sigma_{p+1}+\cdots+\sigma_{n}\right) .
$$

\section{Application to surface acoustic wave driven microfluidic biochips}

We apply the model reduction based optimization of the Stokes system to the optimal design of capillary barriers for surface acoustic wave driven microfluidic biochips. Microfluidic biochips are used in pharmaceutical, medical, and forensic applications for high throughput screening, genotyping, and sequencing in genomics, protein profiling in proteomics, and cytometry in cell analysis [42, 48]. They provide a much better sensitivity and a greater flexibility than traditional approaches. More importantly, they give rise to a significant speed-up of the hybridization processes and allow the in-situ investigation of these processes at an extremely high time resolution. This can be achieved by integrating the fluidics on top of the chip by means of a lithographically produced network of channels and reservoirs (cf. Fig. 1 (left)). The idea is to inject a DNA or protein containing probe and to transport it in the fluid to a reservoir where a chemical analysis is performed. The fluid flow is induced by surface acoustic waves (SAW) generated by interdigital transducers placed on a piezoelectric substrate which allows a much more accurate control of the flow than conventional external pumps $[19,26,53,54,55]$. In order to guarantee the filling of the reservoirs with a precise amount of the probe, pressure driven capillary barriers are placed between the channels and the reservoirs (cf. Fig. 1 (right)). As long as the pressure is above a certain threshold, there is fluid flow from the channel into the reservoir (flow mode). Otherwise, there is no inflow, i.e., the barrier is in the stopping mode. One of the optimization issues is to design the capillary barrier such that the velocity and the pressure in the flow mode is as close as possible to a prescribed velocity and pressure profile.
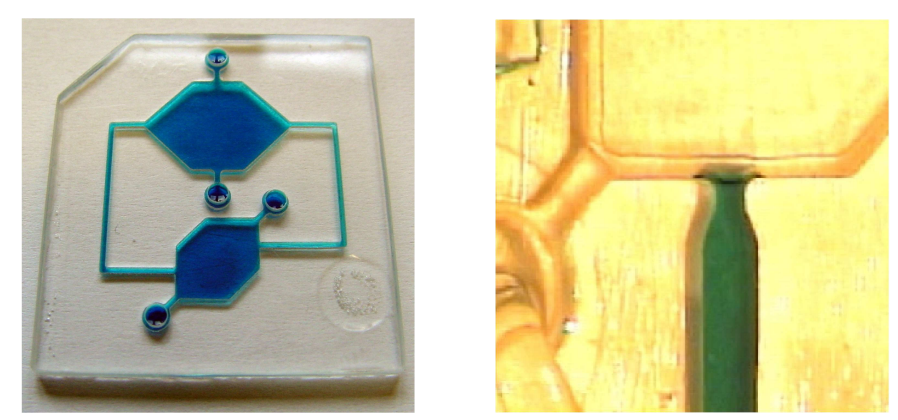

FIGURE 1. Microfluidic biochip (left) and capillary barrier (right) 
SAW driven microfluidic biochips are modeled by a system of PDEs consisting of the linearized equations of piezoelectricity coupled with the compressible NavierStokes equations (see, e.g., $[1,2]$ ). We consider the SAW induced fluid flow in the fluidic network on top of the biochip and denote by $\Omega \subset \mathbb{R}^{2}$ the domain occupied by the fluid. Its boundary is split according to $\Gamma=\bar{\Gamma}_{D} \cup \bar{\Gamma}_{N}, \Gamma_{D} \cap \Gamma_{N}=\emptyset$, where $\Gamma_{D}$ stands for the part where the SAW enter the fluid filled microchannels. We further denote by $v$ and $p$ the velocity and the pressure, and we refer to $\rho, \eta$, and $\xi$ as the density of the fluid and the standard and bulk viscosities. The pair $(v, p)$ satisfies the following initial-boundary value problem

$$
\begin{array}{rlrl}
\rho\left(\frac{\partial v}{\partial t}+v \cdot \nabla v\right) & =\nabla \cdot \sigma & & \text { in } \Omega, t \in(0, T], \\
\frac{\partial \rho}{\partial t}+\nabla \cdot(\rho v) & =0 & & \text { in } \Omega, t \in(0, T], \\
v(\cdot+u(\cdot, t), t) & =\frac{\partial u}{\partial t}(\cdot, t) & & \text { on } \Gamma_{D}, t \in(0, T] \\
\sigma n=0 & & \text { on } \Gamma_{N}, t \in(0, T], \\
v(\cdot, 0)=v_{0}, \quad p(\cdot, 0)=p_{0} & & \text { in } \Omega(0),
\end{array}
$$

where $\sigma=\left(\sigma_{i j}\right)_{i, j=1}^{2}, \sigma_{i j}:=-p \delta_{i j}+2 \eta \varepsilon_{i j}(v)+\delta_{i j}(\xi-2 \eta / 3) \nabla \cdot v$ and $u$ in (6.1c) stands for the deflection of the walls of the microchannels caused by the SAW. We note that $u$ can be computed by the solution of the linearized equations of piezoelectricity (see, e.g., [22]) and that we have neglected the time dependence of the domain, since the deflection of the walls of the microchannels by the SAW (approximately $10^{-9} \mathrm{~m}$ ) is small compared to the lengths, widths, and heights of the microchannels $(\mu m$ to $m m)$.

The SAW induced fluid flow occurs on two different time scales. When the SAW enter the fluid filled microchannels, sharp jets are created which is a process that happens within nanoseconds. Then, the SAW propagate along the channels and experience a significant damping resulting in a flow pattern called acoustic streaming. This relaxation process happens on a time scale of milliseconds. We are thus faced with a multiscale fluid flow which can be appropriately handled by homogenization. Following [1,33], we introduce a scale parameter $0<\varepsilon \ll 1$ and consider the asymptotic expansions

$$
\begin{aligned}
& \rho=\rho_{0}+\varepsilon \rho^{\prime}+\varepsilon^{2} \rho^{\prime \prime}+O\left(\varepsilon^{3}\right) \\
& \mathbf{v}=\mathbf{v}_{0}+\varepsilon \mathbf{v}^{\prime}+\varepsilon^{2} \mathbf{v}^{\prime \prime}+O\left(\varepsilon^{3}\right) \\
& p=p_{0}+\varepsilon p^{\prime}+\varepsilon^{2} p^{\prime \prime}+O\left(\varepsilon^{3}\right)
\end{aligned}
$$

If we collect all terms of order $O(\varepsilon)$, assuming $\mathbf{v}_{0} \equiv 0$ (fluid at rest, if no SAW actuation), and set $\rho_{1}=\varepsilon \rho^{\prime}, v_{1}:=\varepsilon v^{\prime}, p_{1}:=\varepsilon p^{\prime}$, we find that the triple $\left(\rho_{1}, v_{1}, p_{1}\right)$ satisfies the following time-periodic initial-boundary value problem for the linear 
compressible Navier-Stokes equations

$$
\begin{aligned}
\rho_{0} \frac{\partial v_{1}}{\partial t}-\nabla \cdot \sigma_{1} & =0 & & \text { in } \Omega \times\left(0, T_{1}\right], \\
\frac{\partial \rho_{1}}{\partial t}+\rho_{0} \nabla \cdot v_{1} & =0 & & \text { in } \Omega \times\left(0, T_{1}\right], \\
v_{1} & =g_{1} & & \text { on } \Gamma_{D} \times\left(0, T_{1}\right], \\
\sigma_{1} n & =0 & & \text { on } \Gamma_{N} \times\left(0, T_{1}\right], \\
v_{1}(\cdot, 0)=0, \quad p_{1}(\cdot, 0) & =0 & & \text { in } \Omega,
\end{aligned}
$$

where $T_{1}:=2 \pi / \omega$ with $\omega$ being the angular frequency of the time harmonic SAW excitation, $g_{1}:=\partial u / \partial t$ in $(6.2 \mathrm{c})$, and

$$
\sigma_{1}=\left(\left(\sigma_{1}\right)_{i j}\right)_{i, j=1}^{2},\left(\sigma_{1}\right)_{i j}:=-p_{1} \delta_{i j}+2 \eta \varepsilon_{i j}\left(v_{1}\right)+\delta_{i j}(\xi-2 \eta / 3) \nabla \cdot v_{1} .
$$

Moreover, $p_{1}$ and $\rho_{1}$ are related by the constitutive equation $p_{1}=c_{0}^{2} \rho_{1}$ in $\Omega \times$ $\left(0, T_{1}\right]$, where $c_{0}$ stands for the small signal sound speed in the fluid. The system (6.2a)-(6.2e) describes the propagation and damping of the acoustic waves in the microchannels.

Now, we collect all terms of order $O\left(\varepsilon^{2}\right)$, neglect the time derivative with respect to the pressure, and perform the time-averaging $\langle w\rangle:=T_{1}^{-1} \int_{t_{0}}^{t_{0}+T_{1}} w d t$. We thus arrive at the compressible Stokes system

$$
\begin{aligned}
\rho_{0} \frac{\partial v_{2}}{\partial t}-\nabla \cdot \sigma_{2} & =\left\langle-\rho_{1} \frac{\partial v_{1}}{\partial t}-\rho_{0}\left[\nabla v_{1}\right] v_{1}\right\rangle & & \text { in } \Omega \times(0, T], \\
\rho_{0} \nabla \cdot v_{2} & =\left\langle-\nabla \cdot\left(\rho_{1} v_{1}\right)\right\rangle & & \text { in } \Omega \times(0, T], \\
v_{2} & =g_{2} & & \text { on } \Gamma_{D} \times(0, T], \\
\sigma_{2} n & =0 & & \text { on } \Gamma_{N} \times(0, T], \\
v_{2}(\cdot, 0) & =0, p_{2}(\cdot, 0)=0 & & \text { in } \Omega,
\end{aligned}
$$

where $g_{2}:=-\left\langle\left[\nabla v_{1}\right] u\right\rangle$ in $(6.3 \mathrm{c})$ and

$$
\sigma_{2}=\left(\left(\sigma_{2}\right)_{i j}\right)_{i, j=1}^{2},\left(\sigma_{2}\right)_{i j}:=-p_{2} \delta_{i j}+2 \eta \varepsilon_{i j}\left(v_{2}\right)+\delta_{i j}(\xi-2 \eta / 3) \nabla \cdot v_{2} .
$$

The density $\rho_{2}$ can be obtained via the constitutive equation $p_{2}=c_{0}^{2} \rho_{2}$ in $\Omega \times$ $(0, T]$. We use the compressible Stokes system (6.3a)-(6.3e) as a model for the acoustic streaming.

For the optimal design of a capillary barrier, we consider acoustic streaming as described by (6.3a)-(6.3e) in a network of microchannels and reservoirs on top of a microfluidic biochip with a capillary barrier between a channel and a reservoir (cf. Figure 2). The computational domain $\Omega$ is decomposed into subdomains $\Omega_{1}=$ $\Omega \backslash \Omega_{2}$, and $\Omega_{2}=(1.5,2.5) \times(9,10) \mathrm{mm}^{2}$. The boundary $\partial \Omega$ is split into $\Gamma_{\text {in }}=$ $\{0\} \times(9.4,10), \Gamma_{\text {out }}=\{10\} \times(0,1) \mathrm{mm}^{2}$, and $\Gamma_{\text {lat }}=\partial \Omega \backslash\left(\Gamma_{\text {in }} \cup \Gamma_{\text {out }}\right)$. We assume that an interdigital transducer of width $6 \mathrm{~mm}$ is placed at $\Gamma_{\text {in }}$ and that the input 

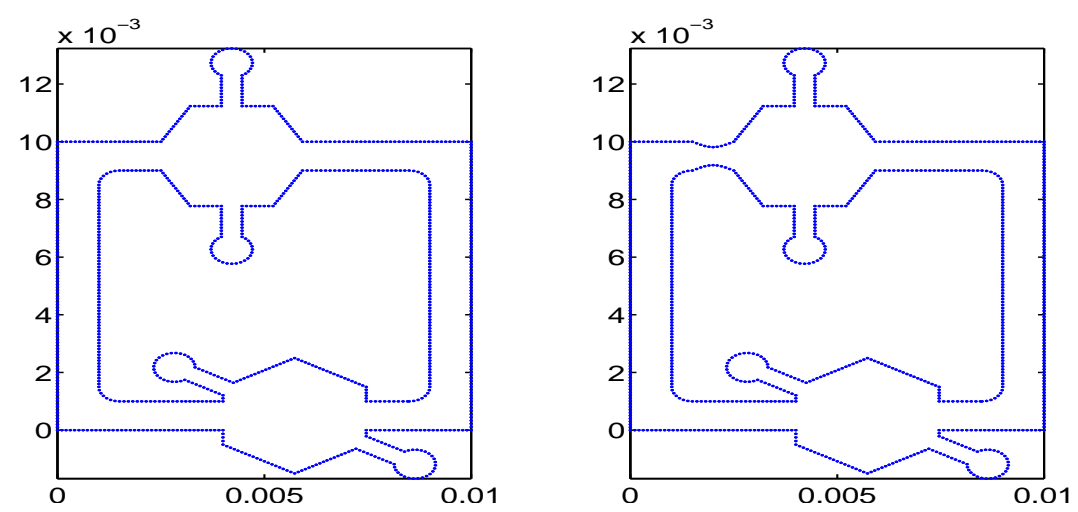

Figure 2. Optimal design of a capillary barrier: The reference domain $\Omega_{\text {ref }}($ left, in $[\mathrm{m}]$ ) and the optimal domain (right, in $[\mathrm{m}]$ ).

velocity profile $u=\left(u_{1}, u_{2}\right)$ is given by

$$
\begin{aligned}
& u_{1}\left(t, x_{1}\right)=0.6 \epsilon \sin \left(2 \pi\left(-\hat{k} x_{1}+f t\right)\right), \\
& u_{2}\left(t, x_{1}\right)=-\epsilon \cos \left(2 \pi\left(-\hat{k} x_{1}+f t\right)\right)
\end{aligned}
$$

with appropriately chosen constants $\epsilon, \hat{k}$ and $f$. We further choose a constant velocity profile $v_{\text {in }}\left(x_{1}, x_{2}\right)$ on $\Gamma_{\text {in }} \times(0, T)$, outflow boundary conditions on $\Gamma_{\text {out }} \times$ $(0, T)$, and no-slip conditions on $\Gamma_{\text {lat }} \times(0, T)$. The objective is to design the shape of the top $\Gamma_{2, T}$ and the bottom $\Gamma_{2, B}$ of $\partial \Omega_{2}$ in such a way that a prescribed velocity profile $\mathbf{v}^{d}$ is achieved in $\Omega_{2} \times(0, T)$ and that the vorticity is minimized in $\Omega_{\text {obs }}$ (the two bulb shaped structures associated with the lower reservoir in Figure 2). The subdomain $\Omega_{2}$ is parameterized by representing the top and bottom boundary by Bézier curves with $d_{t o p}=6$ and $d_{b o t}=6$ control points, respectively. This leads to a parametrization $\Omega_{2}(\theta)$ of $\Omega_{2}$ with parameters $\theta \in \mathbb{R}^{d_{t o p}+d_{b o t}}$.

The shape optimization problem amounts to the minimization of

$$
J(\theta)=\int_{0}^{T} \int_{\Omega_{\mathrm{obs}}}|\nabla \times v(x, t)|^{2} d x d t+\int_{0}^{T} \int_{\Omega_{2}(\theta)}\left|v(x, t)-v^{d}(x, t)\right|^{2} d x d t
$$

subject to (6.3a)-(6.3e) and the design parameter constraints

$$
\theta^{\min } \leq \theta \leq \theta^{\max } \text {. }
$$

The final time $T$ is $T=0.1 \mathrm{~ms}$, and the bounds $\theta^{\min }, \theta^{\max }$ on the design parameters are chosen such that the design constraints are never active in this example. The optimal domain $\Omega\left(\theta^{*}\right)$ is shown in Figure 2 (right).

We consider a geometrically conforming simplicial triangulation $\mathcal{T}_{h}(\Omega)$ of $\Omega$ that aligns with the decomposition into the subdomains $\Omega_{1}$ and $\Omega_{2}$ as well as the 
respective boundaries. The semi-discretization in space is performed by TaylorHood P2-P1 elements. We denote by $N_{v}^{(1)}, N_{v}^{(2)}, N_{v}^{\Gamma}$ the number of velocity degrees of freedom in the subdomains $\bar{\Omega}_{1} \backslash \Gamma, \bar{\Omega}_{2} \backslash \Gamma$ and in $\Gamma$, respectively, and set $N_{v}=N_{v}^{(1)}+N_{v}^{(2)}+N_{v}^{\Gamma}$. Similarly, $N_{p}^{(1)}, N_{p}^{(2)}$ stand for the numbers of pressure degrees of freedom in the subdomains $\bar{\Omega}_{1}, \bar{\Omega}_{2}$ and $N_{p}=N_{p}^{(1)}+N_{p}^{(2)}$ is the total number of pressure degrees of freedom.

The semi-discretized optimization problems is solved by a projected BFGS method with Armijo line search [32], and the optimization algorithm is terminated when the norm of the projected gradient is less than $2 \cdot 10^{-8}$. We use automatic differentiation $[25,46]$ to compute the derivatives with respect to the design variables.

We have applied the combination of domain decomposition and balanced truncation model reduction (DDBTMR) to the semi-discretized Stokes system (6.3a)-(6.3e) using four different finite element meshes. Figure 3 (left) displays the convergence of the multi-shift ADI algorithm for the computation of the controllability Gramian $\mathbf{P}$ and the observability Gramian $\mathbf{Q}$, and Figure 3 (right) shows the computed Hankel singular values for the finest grid problem. The constant $C$ in the estimate (5.10) for the error between the optimal design parameters, computed by the full and the reduced order problems, depends on quantities like $\alpha$ in (2.3) of Theorem 2.1, the derivatives of $\mathbf{A}(\theta)$ with respect to $\theta$, etc. Numerical experiments indicate that for the current scaling of the problem, the constant $C$ in the estimate (5.10) is large. Therefore, we require a rather small truncation level of $\sigma_{p+1}<10^{-12} \sigma_{1}$ for the Hankel singular values.
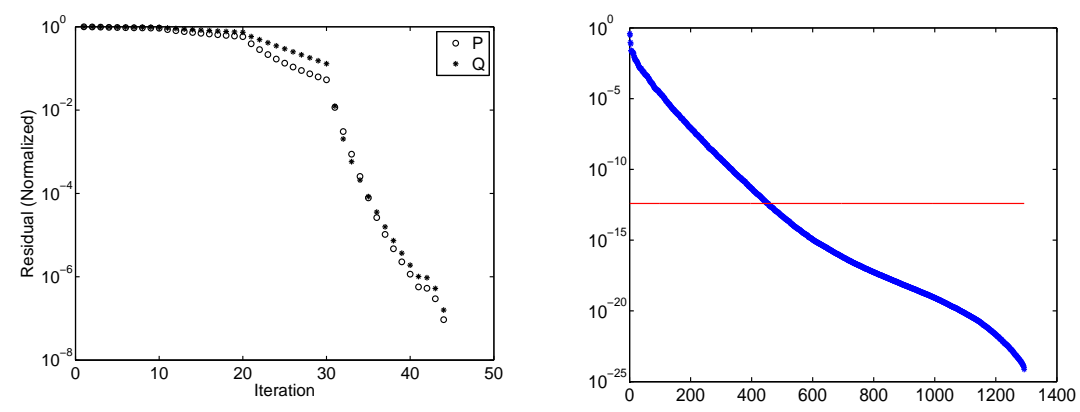

Figure 3. Convergence of the multishift ADI (left); the largest Hankel singular values and the threshold $10^{-12} \sigma_{1}$ (right).

Table 1 shows the sizes $N_{v}^{(1)}, N_{v}$ of the full order models on the four grids as well as the sizes $N_{\widehat{v}}^{(1)}, N_{\widehat{v}}$ of the reduced order models in subdomain $\Omega_{1}$ and in $\Omega$. For the finest grid, DDBTMR reduced the size of the $\Omega_{1}$ subproblem from $N_{v}^{(1)}=48324$ to $N_{\widehat{v}}^{(1)}=766$. The velocity degrees of freedom in $\Omega_{2} \cup \Gamma$ are not reduced. On the finest grid these are $N_{v}^{(2)}+N_{v}^{\Gamma}=914$. Therefore, the reduced order problem has $N_{\widehat{v}}=914+766=1680$ degrees of freedom. 


\begin{tabular}{|c|c|c|c|c|c|}
\hline grid & $m$ & $N_{v}^{(1)}$ & $N_{\widehat{v}}^{(1)}$ & $N_{v}$ & $N_{\widehat{v}}$ \\
\hline 1 & 167 & 7482 & 351 & 7640 & 509 \\
2 & 195 & 11442 & 370 & 11668 & 596 \\
3 & 291 & 16504 & 451 & 16830 & 777 \\
4 & 802 & 48324 & 766 & 49238 & 1680 \\
\hline
\end{tabular}

TABLE 1. The number of $m$ of observations, the numbers $N_{v}^{(1)}$, $N_{v}$ of velocity degrees of freedom in subdomain $\Omega_{1}$ and in $\Omega$ for the full order model, and the numbers $N_{\widehat{v}}^{(1)}, N_{\widehat{v}}$ of velocity degrees of freedom in subdomain $\Omega_{1}$ and in $\Omega$ for the reduced order model.

The optimal shape parameters $\theta^{*}$ and $\widehat{\theta}^{*}$ computed by minimizing the full and the reduced order model, respectively, are shown in Table 2. For the finest grid, the error between the full and the reduced order model solutions is $\left\|\theta^{*}-\widehat{\theta}^{*}\right\|=$ $3.9165 \cdot 10^{-5}$.

$$
\begin{array}{|c|c|}
\hline \theta^{*} & (9.8833,9.7467,9.7572,9.8671,9.1336,9.2015,9.1971,9.1310) \times 10^{-3} \\
\widehat{\theta}^{*} & (9.8694,9.7374,9.7525,9.8628,9.1498,9.2044,9.1895,9.1204) \times 10^{-3} \\
\hline
\end{array}
$$

TABLE 2. Optimal shape parameters $\theta^{*}$ and $\widehat{\theta}^{*}$ computed by minimizing the full and the reduced order model.

Acknowledgement. The results presented in this paper have been obtained in cooperation with Harbir Antil and Matthias Heinkenschloss from Rice University. We are thankful to our colleagues for their most valuable contributions.

\section{References}

[1] H. Antil, A. Gantner, R.H.W. Hoppe, D. Köster, K. G. Siebert, and A. Wixforth, Modeling and simulation of piezoelectrically agitated acoustic streaming on microfluidic biochips, in Domain Decomposition Methods in Science and Engineering XVII, U. Langer et al., eds., Lecture Notes in Computational Science and Engineering, Vol. 60, Springer, Berlin-Heidelberg-New York, 2008, pp. 305-312.

[2] H. Antil, R. Glowinski, R.H.W. Hoppe, C. Linsenmann, T.W. Pan, and A. Wixforth, Modeling, simulation, and optimization of surface acoustic wave driven microfluidic biochips. J. Comp. Math. 28, 149-169, 2010.

[3] H. Antil, M. Heinkenschloss, R. H. W. Hoppe, and D.C. Sorensen, Domain decomposition and model reduction for the numerical solution of PDE constrained optimization problems with localized optimization variables. Submitted to Comput. Vis. Sci., 2009.

[4] H. Antil, M. Heinkenschloss, and R. H. W. Hoppe, Domain decomposition and balanced truncation model reduction for shape optimization of the Stokes system. Submitted to Optimization Methods \& Software, 2009. 
[5] H. Antil, R.H.W. Hoppe, and C. Linsenmann, Path-following primal-dual interiorpoint methods for shape optimization of stationary flow problems. J. Numer. Math. 11, 81-100, 2007.

[6] H. Antil, R.H.W. Hoppe, and C. Linsenmann, Adaptive path following primal dual interior point methods for shape optimization of linear and nonlinear Stokes flow problems. In: Lecture Notes in Computer Science, Vol. 4818, pp. 259-266, Springer, Berlin-Heidelberg-New York, 2008.

[7] H. Antil, R.H.W. Hoppe, and C. Linsenmann, Optimal design of stationary flow problems by path-following interior-point methods. Control and Cybernetics 37, 771796, 2008.

[8] A. C. Antoulas, Approximation of Large-Scale Systems. SIAM, Philadelphia, 2005.

[9] A. C. Antoulas, M. Heinkenschloss, and T. Reis, On balanced truncation for inhomogeneously initialized systems. Technical Report TR09-29, Department of Computational and Applied Mathematics, Rice University, 2009.

[10] Z. Bai, P. Dewilde, and R. Freund, Reduced order modeling. Handbook of Numerical Analysis, Vol. XIII (W. Schilders and E.J.W. ter Maten; eds.), pp. 825-895, NorthHolland/Elsevier, Amsterdam, 2005.

[11] P. Benner, R.W. Freund, D. Sorensen, and A. Varga (eds.), Special issue on 'order reduction of large-scale systems'. Linear Algebra and its Applications 415 (2-3), 2006.

[12] P. Benner, V. Mehrmann, and D. C. Sorensen (eds.), Dimension Reduction of LargeScale Systems. Lecture Notes in Computational Science and Engineering, Vol. 45, Springer, Berlin-Heidelberg-New York, 2005.

[13] D. Braess, Finite elements. Theory, Fast Solvers, and Applications in Elasticity Theory. 3rd Ed.. Cambridge University Press, Cambridge, 2007.

[14] F. Brezzi and M. Fortin, Mixed and Hybrid Finite Element Methods. Springer, BerlinHeidelberg-New York, 1991.

[15] T. Bui-Thanh, K. Willcox, and O. Ghattas, Model reduction for large-scale systems with high-dimensional parametric input space. SIAM J. Sci. Comp. 30, 3270-3288, 2008.

[16] T. Bui-Thanh, K. Willcox, O. Ghattas, and B. van Bloemen Wanders, Goal-oriented, model-constrained optimization for reduction of large-scale systems. J. Comp. Phys. 224, 880-896, 2007.

[17] M. Fahl and E. Sachs, Reduced order modelling approaches to PDE-constrained optimization based on proper orthogonal decomposition. In: Large-Scale PDEConstrained Optimization (L.T. Biegler et al.; eds.), Lect. Notes in Comput. Sci. Engrg., Vol. 30, Springer, Berlin-Heidelberg-New York, 2003.

[18] M.A. Fallah, SAW induced acoustic streaming in microchannels of different geometry. Master's Thesis. Institute of Physics, University of Augsburg, 2008.

[19] T. Franke and A. Wixforth, Microfluidics for miniaturized laboratories on a chip. ChemPhysChem 9, 2140-2156, 2008.

[20] R.W. Freund, Model reduction methods based on Krylov subspaces. Acta Numerica, 267-319, 2003.

[21] V. Girault and P.-A. Raviart, Finite Element Methods for Navier-Stokes Equations. Theory and Algorithms. Springer, Berlin-Heidelberg-New York, 1986. 
[22] A. Gantner, R.H.W. Hoppe, D. Köster, K.G. Siebert, and A. Wixforth, Numerical simulation of piezoelectrically agitated surface acoustic waves on microfluidic biochips, Comp. Visual. Sci. 10, 145-161, 2007.

[23] M.A. Grepl and A.T. Patera, A posteriori error bounds for reduced-basis approximations of parametrized parabolic partial differential equations. ESAIM: M2AN, 39, 157-181, 2005.

[24] M.A. Grepl, Y. Maday, N.C. Nguyen, and A.T. Patera, Efficient reduced-basis treatment of nonaffine and nonlinear partial differential equations. ESAIM: M2AN, 41, 575-605, 2007.

[25] A. Griewank and A. Walther, Evaluating Derivatives. Principles and Techniques of Algorithmic Differentiation. 2nd Ed.. SIAM, Philadelphia, 2008.

[26] Z. Guttenberg, H. Muller, H. Habermuller, A. Geisbauer, J. Pipper, J. Felbel, M. Kielpinski, J. Scriba, and A. Wixforth, Planar chip device for PCR and hybridization with surface acoustic wave pump. Lab Chip 5, 308-317, 2005.

[27] B. Haasdonk, M. Ohlberger, and G. Rozza, A reduced basis method for evolution schemes with parameter-dependent explicit operators. Electronic Transactions on $\mathrm{Nu}-$ merical Analysis 32, 145-161, 2008.

[28] M. Heinkenschloss, D. C. Sorensen, and K. Sun, Balanced truncation model reduction for a class of descriptor systems with application to the Oseen equations. SIAM Journal on Scientific Computing, 30, 1038-1063, 2008.

[29] M. Hinze and S. Volkwein, Proper orthogonal decomposition surrogate models for nonlinear dynamical systems: Error estimates and suboptimal control. In: Dimension Reduction of Large-Scale Systems (P. Benner, V. Mehrmann, and D.S. Sorensen; eds.), pages 261-306, Lecture Notes in Computational Science and Engineering, Vol. 45, Springer, Berlin-Heidelberg-New York, 2005.

[30] M. Hinze and S. Volkwein, Error estimates for abstract linear-quadratic optimal control problems using proper orthogonal decomposition. Comp. Optim. Appl. 39, 319-345, 2008.

[31] K. Ito and S.S. Ravindran, A reduced order method for simulation and control of fluid flows. J. Comp. Phys. 143, 403-425, 1998.

[32] C. T. Kelley, Iterative Methods for Optimization. SIAM, Philadelphia, 1999.

[33] D. Köster, Numerical simulation of acoustic streaming on SAW-driven biochips, SIAM J. Comp. Sci. 29, 2352-2380, 2007.

[34] K. Kunisch and S. Volkwein, Galerkin proper orthogonal decomposition methods for parabolic problems. Numer. Math. 90, 117-148, 2001.

[35] K. Kunisch and S. Volkwein, Galerkin proper orthogonal decomposition methods for a general equation in fluid dynamics. SIAM J. Numer. Anal. 40, 492-515, 2002.

[36] K. Kunisch and S. Volkwein, Proper orthogonal decomposition for optimality systems. M2AN, 42, 1-23, 2008.

[37] J.L. Lions and E. Magenes, Non-homogeneous Boundary Value Problems and Applications. Vol. I,II. Springer, Berlin-Heidelberg-New York, 1972.

[38] H.V. Ly and H.T. Tran, Modelling and control of physical processes using proper orthogonal decomposition. Math. and Comput. Modelling 33, 223-236, 2001. 
[39] H.V. Ly and H.T. Tran, Proper orthogonal decomposition for flow calculations and optimal control in a horizontal CVD reactor. Quart. Appl. Math. 60, 631-656, 2002.

[40] V. Mehrmann and T. Stykel, Balanced truncation model reduction for large-scale systems in descriptor form. In: Dimension Reduction of Large-Scale Systems (P. Benner, V. Mehrmann, and D. C. Sorensen; eds.), Lecture Notes in Computational Science and Engineering, Vol. 45, pp. 83-115, Springer, Berlin-Heidelberg-New York, 2005.

[41] A.T. Patera and G. Rozza, Reduced Basis Methods and A Posteriori Error Estimation for Parametrized Partial Differential Equations. MIT Pappalardo Graduate Monograqphs in Mechanical Engineering, MIT, Boston, 2007.

[42] J. Pollard and B. Castrodale, Outlook for DNA microarrays: emerging applications and insights on optimizing microarray studies, Report. Cambridge Health Institute, Cambridge 2003.

[43] T. Reis and M. Heinkenschloss, Model reduction with a-priori error bounds for a class of nonlinear electrical circuits. To appear in: Proc. 48th IEEE Conference on Decision and Control, Shanghai, P.R. China, December 16-18, 2009.

[44] C. W. Rowley, Model reduction for fluids, using balanced proper orthogonal decomposition. Int. J. on Bifurcation and Chaos 15, 997-1013, 2005.

[45] C.W. Rowley, T. Colonius, and R.M. Murray, Model reduction for compressible fluids using POD and Galerkin projection. Phys. D 189, 115-129, 2004.

[46] S. M. Rump, INTLAB - INTerval LABoratory. In: Tibor Csendes, editor, Developments in Reliable Computing, pages 77-104. Kluwer Academic Publishers, Dordrecht, 1999.

[47] W.H.A. Schilders, H.A. van der Vorst, and J. Rommes (eds.), Model Order Reduction for MEMS: Methodology and Computational Environment for Electro-Thermal Models. Mathematics in Industry, Vol. 13, Springer, Berlin-Heidelberg-NewYork, 2008.

[48] H.M. Shapiro, Practical flow cytometry. Wiley-Liss, New York, 2003.

[49] T. Stykel, Balanced truncation model reduction for semidiscretized Stokes equation. Linear Algebra Appl. 415, 262-289, 2006.

[50] F. Tröltzsch and S. Volkwein, POD a posteriori error estimates for linear-quadratic optimal control problems. Comp. Opt. and Appl. 44, 83-115, 2009.

[51] S. Volkwein, Optimal control of a phase-field model using proper orthogonal decomposition. ZAMM 81, 83-97, 2001.

[52] K. Willcox and J. Peraire, Balanced model reduction via the proper orthogonal decomposition. AIAA 40, 2323-2330, 2002.

[53] A. Wixforth, Acoustically driven programmable microfluidics for biological and chemical applications. JALA 11, 399-405, 2006.

[54] A. Wixforth, J. Scriba, and G. Gauer, Flatland fluidics. mst news 5, 42-43, 2002.

[55] L.Y. Yeo and J.R. Friend, Ultrafast microfluidics using surface acoustic waves. Biomicrofluidics 3, 012002-012023, 2009. 


\section{T. Franke}

Institute of Physics

Universität Augsburg

D-86159 Augsburg

Germany

e-mail: franketh@physik.uni-augsburg.de

R.H.W. Hoppe

Institute of Mathematics

Universität Augsburg

D-86159 Augsburg

Germany

Department of Mathematics

University of Houston

Houston, TX 77204-3008

USA

e-mail: hoppe@math.uni-augsburg.de, rohop@math.uh.edu

C. Linsenmann

Institute of Mathematics

Universität Augsburg

D-86159 Augsburg

Germany

e-mail: christopher.linsenmann@math.uni-augsburg.de

A. Wixforth

Institute of Physics

Universität Augsburg

D-86159 Augsburg

Germany

e-mail: achim.wixforth@physik.uni-augsburg.de 